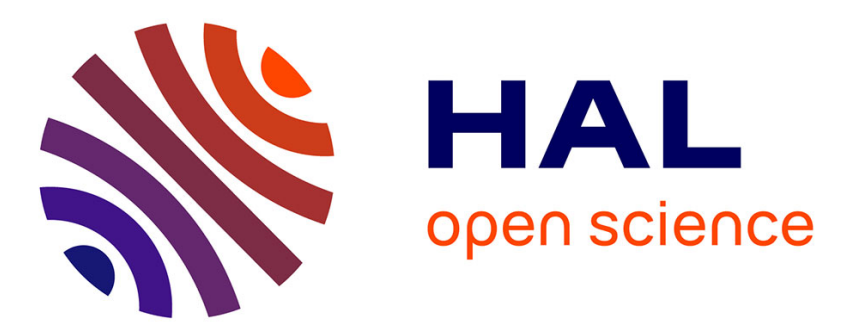

\title{
The geometry of the Coble cubic and orbital degeneracy loci
}

\author{
Vladimiro Benedetti, Laurent Manivel, Fabio Tanturri
}

\section{To cite this version:}

Vladimiro Benedetti, Laurent Manivel, Fabio Tanturri. The geometry of the Coble cubic and orbital degeneracy loci. Mathematische Annalen, 2021, 379 (1-2), pp.415-440. 10.1007/s00208-019-01949-7 . hal-02110007

\section{HAL Id: hal-02110007 https://hal.science/hal-02110007}

Submitted on 25 Apr 2019

HAL is a multi-disciplinary open access archive for the deposit and dissemination of scientific research documents, whether they are published or not. The documents may come from teaching and research institutions in France or abroad, or from public or private research centers.
L'archive ouverte pluridisciplinaire HAL, est destinée au dépôt et à la diffusion de documents scientifiques de niveau recherche, publiés ou non, émanant des établissements d'enseignement et de recherche français ou étrangers, des laboratoires publics ou privés. 


\title{
The geometry of the Coble cubic and orbital degeneracy loci
}

\author{
Vladimiro Benedetti, Laurent Manivel’, Fabio Tanturri ${ }^{\ddagger}$
}

\begin{abstract}
The Coble cubics were discovered more than a century ago in connection with genus two Riemann surfaces and theta functions. They have attracted renewed interest ever since. Recently, they were reinterpreted in terms of alternating trivectors in nine variables. Exploring this relation further, we show how the Hilbert scheme of pairs of points on an abelian surface, and also its Kummer fourfold, a very remarkable hyper-Kähler manifold, can very naturally be constructed in this context. Moreover, we explain how this perspective allows us to describe the group law of an abelian surface, in a strikingly similar way to how the group structure of a plane cubic can be defined in terms of its intersection with lines.
\end{abstract}

\section{Introduction}

The Coble hypersurfaces are very remarkable cubics and quartics in complex projective spaces, discovered by Coble more than a century ago. They can be characterized as the unique hypersurfaces whose singular locus is the Jacobian of a genus two curve embedded in $\mathbf{P}^{8}$, or the associated Kummer variety of a genus three curve embedded in $\mathbf{P}^{7}$, respectively.

The Coble hypersurfaces have been revisited several times. In the eighties, Narasimhan and Ramanan interpreted them in terms of moduli spaces of vector bundles with fixed determinant on a curve of genus two or three [NR87]. This perspective has been explored by a number of authors, see [Bea03] and the references therein.

More recently, the Coble hypersurfaces have been given interpretations coming from Lie theory, more precisely from the Kac-Vinberg theory of so-called $\theta$-groups [GSW13]. It seems to be just a coincidence that $\theta$-groups were coined this way at a time where there was no apparent relation with theta functions, but the fact is that there is a very rich interplay between the invariant theory of $\theta$-representations and certain moduli spaces of polarized abelian varieties.

From this point of view, genus two curves are naturally related to alternating trivectors, that is, elements of $\wedge^{3} V_{9}$ for $V_{9}$ a nine-dimensional vector space. Over the complex numbers, it was explained in [GSW13] how to associate to a general

\footnotetext{
*Département de mathématiques et applications, ENS, CNRS, PSL University, 75005 Paris, France

${ }^{\dagger}$ Institut de Mathématiques de Toulouse, UMR 5219, Université de Toulouse, CNRS, UPS IMT F-31062 Toulouse Cedex 9, France.

${ }^{\ddagger}$ Laboratoire Paul Painlevé, UMR CNRS 8524, Université de Lille, 59655 Villeneuve d’Ascq CEDEX, France.
} 
such trivector an abelian surface $A$ in $\mathbf{P}\left(V_{9}^{\vee}\right)$, the projective space of hyperplanes in $V_{9}$, and a cubic hypersurface which is singular exactly along $A$; to be precise, $A$ is only a torsor over an abelian surface: to make it an abelian surface stricto sensu, one needs to fix an origin. Of course this cubic hypersurface has to be the same as the one discovered by Coble. This point of view was further explored in [GS15] and, over an arbitrary field, in [RS18].

In this paper we shall enrich the picture by passing to the dual projective space $\mathbf{P}\left(V_{9}\right)$, where our trivector defines a wealth of interesting subvarieties, that we interpret as orbital degeneracy loci in Section 4, following the terminology of [BFMT17, BFMT18]. Already well-known were the projective dual to the cubic, which is a special sextic hypersurface, and a (singular) subvariety $\Sigma$ of its singular locus which can be identified with the triples of degree zero line bundles on the genus two curve whose product is trivial. A nice ingredient from the theory of orbital degeneracy loci is that they usually come with simple resolutions of singularities, just like the usual degeneracy loci of morphisms between vector bundles. We show that our natural resolution of $\Sigma$ is nothing else than the Kummer fourfold of $A$ (Theorem 5.1). Moreover, we observe that from the orbital degeneracy loci point of view, one can define two natural smooth covers of $\Sigma$, generically finite of degree three. We identify these covers, one with the Hilbert scheme of length two subschemes of $A$ (Theorem 5.14), the other one with the nested Kummer fourfold (Theorem 5.11). More precisely, the main results can be summarized in the following theorem, where we denoted by $\mathcal{U}_{r_{i}}$ the tautological bundle of rank $r_{i}$ over the flag variety $F\left(r_{1}, \ldots, r_{i}, \ldots, V_{9}\right)$.

Theorem. Let $\omega \in \wedge^{3} V_{9}$ be a general alternating trivector and let $A$ be its associated abelian surface in $\mathbf{P}\left(V_{9}^{\vee}\right)$. If we regard $\omega$ as a general section of the trivial vector bundle $\wedge^{3} V_{9}$ over $\mathbf{P}\left(V_{9}\right)$, then:

- the zero locus of the section induced by $\omega$ of the vector bundle

$$
\pi^{*}\left(\wedge^{3} V_{9}\right) /\left(\mathcal{U}_{1} \wedge \wedge^{2} V_{9}+\mathcal{U}_{3} \wedge \mathcal{U}_{6} \wedge V_{9}+\wedge^{3} \mathcal{U}_{6}\right)
$$

over the flag variety $F\left(1,3,6, V_{9}\right) \stackrel{\pi}{\rightarrow} \mathbf{P}\left(V_{9}\right)$ is isomorphic to the generalized Kummer fourfold $\operatorname{Kum}^{2}(A)$;

- the zero locus of the section induced by $\omega$ of the vector bundle

$$
\pi^{*}\left(\wedge^{3} V_{9}\right) /\left(\mathcal{U}_{1} \wedge \wedge^{2} V_{9}+\wedge^{2} \mathcal{U}_{5} \wedge V_{9}+\wedge^{3} \mathcal{U}_{7}\right)
$$

over the flag variety $F\left(1,5,7, V_{9}\right) \stackrel{\pi}{\rightarrow} \mathbf{P}\left(V_{9}\right)$ is isomorphic to the Hilbert scheme $\operatorname{Hilb}^{2}(A)$;

- the zero locus of the section induced by $\omega$ of the vector bundle

$$
\pi^{*}\left(\wedge^{3} V_{9}\right) /\left(\mathcal{U}_{1} \wedge \wedge^{2} V_{9}+\mathcal{U}_{3} \wedge \mathcal{U}_{5} \wedge V_{9}+\mathcal{U}_{3} \wedge \mathcal{U}_{6} \wedge \mathcal{U}_{7}+\wedge^{3} \mathcal{U}_{6}\right)
$$

over the flag variety $F\left(1,3,5,6,7, V_{9}\right) \stackrel{\pi}{\rightarrow} \mathbf{P}\left(V_{9}\right)$ is isomorphic to the nested Kummer fourfold $\mathrm{Kum}^{2,3}(A)$.

On the one hand, the above constructions provide to interesting and wellstudied objects such as $\operatorname{Kum}^{2}(A)$ and $\operatorname{Hilb}^{2}(A)$ an interpretation as zero loci of sections of suitable vector bundles over some flag varieties. On the other hand, this perspective allows us to give the following nice description of the group structure on $A$ (defined once an origin $O$ has been fixed): 
Proposition (Proposition 6.1). Let $A \subset \mathbf{P}\left(V_{9}^{\vee}\right)$ be the abelian surface associated to a general alternating trivector $\omega \in \wedge^{3} V_{9}$. Then we can fix the origin $O$ in $A$ in such a way that the following holds: for any three general points $P, Q, R \in A \subset \mathbf{P}\left(V_{9}^{\vee}\right), P+Q+R=O$ if and only if contracting $\omega$ with any two of the three points yields the same line in $V_{9}$, i.e., if and only if

$$
[\omega(P, Q, \cdot)]=[\omega(P, R, \cdot)]=[\omega(Q, R, \cdot)] \in \mathbf{P}\left(V_{9}\right) .
$$

This description is, at least formally, completely similar to the classical description of the group structure over a plane cubic, from its intersection with lines. The main difference is that the space of "lines", rather than the dual projective plane, is now the Kummer fourfold itself.

Acknowledgements. The authors wish to thank Laurent Gruson for stimulating discussions, and Jerzy Weyman for communicating [KW] to them.

The third author is supported by the Labex CEMPI (ANR-11-LABX-0007-01).

\section{Classical facts}

\subsection{The Coble cubic}

Let $A$ be an abelian surface, and $\Theta$ a principal polarization. Then $3 \Theta$ defines an embedding of $A$ inside $|3 \Theta|^{\vee}=\mathbf{P}\left(V_{9}^{\vee}\right) \simeq \mathbf{P}^{8}$, where $V_{9}:=\mathrm{H}^{0}(A, 3 \Theta)$. The following result is essentially due to Coble [Cob17]:

Theorem 2.1. There exists a unique cubic hypersurface $\mathcal{C}_{3}$ in $\mathbf{P}\left(V_{9}^{\vee}\right)$ which is singular along $A$.

Proof. See, e.g., [Bea03, Proposition 3.1].

We will refer to $\mathcal{C}_{3}$ as the Coble cubic. Note that $A[3]$, the finite group of three-torsion points in $A$, fixes $3 \Theta$. Therefore it acts on $\mathbf{P}\left(V_{9}^{\vee}\right)$ by leaving $A$ invariant.

\subsection{Moduli of vector bundles on genus two curves}

Let $C$ be a genus two curve whose Jacobian $J C \cong A$. Let $\mathrm{SU}_{C}(r)$ denote the moduli space of semistable rank $r$ vector bundles on $C$ with trivial determinant. There is a natural morphism from $\mathrm{SU}_{C}(r)$ to the linear system $|r \Theta|^{\vee}$ (see [Ort05]):

- if $r=2$, we get an isomorphism $\mathrm{SU}_{C}(2) \cong \mathbf{P}^{3}$;

- if $r=3$, we get a finite morphism of degree two $\mathrm{SU}_{C}(3) \rightarrow|3 \Theta|^{\vee}=\mathbf{P}\left(V_{9}\right)$, branched along a sextic hypersurface $\mathcal{C}_{6} \subset \mathbf{P}\left(V_{9}\right)$.

The following result was conjectured by Dolgachev, and proved in [Ort05] and [Ngu07]:

Theorem 2.2. The sextic hypersurface $\mathcal{C}_{6}$ is the projective dual of the Coble cubic $\mathcal{C}_{3}$. 
Remark 2.3. The singular locus of $\mathcal{C}_{6}$ is the same as $\operatorname{Sing}\left(\mathrm{SU}_{C}(3)\right)$, and can be identified with the set of strictly semistable vector bundles on $C$. Its dimension is five. Let $A^{(3)}:=\mathrm{Sym}^{3} A$, let $\sigma: A^{(3)} \rightarrow A$ be the sum morphism, and $\Sigma$ the zero fiber. Then

$$
\Sigma \cong\left\{E \in \mathrm{SU}_{C}(3) \text { s.t. } E=L_{1} \oplus L_{2} \oplus L_{3} \text { with } L_{1}, L_{2}, L_{3} \in J C\right\}
$$

is contained inside $\operatorname{Sing}\left(\operatorname{SU}_{C}(3)\right) \cong \operatorname{Sing}\left(\mathcal{C}_{6}\right) \subset \mathcal{C}_{6} \subset \mathbf{P}\left(V_{9}\right)$.

\subsection{Alternating trivectors}

Following [GSW13], one can give another description of the embedding of $A$ in $\mathbf{P}\left(V_{9}^{\vee}\right)$, starting from an alternating trivector (or three-form).

Let $\omega \in \wedge^{3} V_{9}$ be a general alternating trivector. Let $H$ denote the hyperplane bundle on $\mathbf{P}\left(V_{9}^{\vee}\right)$. Then $\wedge^{3} H$ is a subbundle of the trivial bundle with fiber $\wedge^{3} V_{9}$, and the quotient is $\wedge^{2} H(1)$. So $\omega$ defines a section of $\wedge^{2} H(1)$ over $\mathbf{P}\left(V_{9}^{\vee}\right)$, and the latter can be stratified by the rank of this two-form. We denote

$$
D_{Y_{r}^{S p}}:=\left\{P \in \mathbf{P}\left(V_{9}^{\vee}\right) \text { s.t. } \operatorname{rank}(\omega(P, \cdot, \cdot)) \leq r\right\} .
$$

These loci are nothing more than the degeneracy loci (or Pfaffian loci) of the skew-symmetric morphism $H^{\vee} \rightarrow H(1)$ corresponding to $\omega$. For dimensional reasons, $D_{Y_{2}^{S p}}$ is empty, and therefore $D_{Y_{4}^{S p}}$ is a smooth surface. By [GSW13, Theorem 5.5], $D_{Y_{4}^{S p}}$ is a torsor, that we denote by $A$, over an abelian surface. By [GSW13, Proposition 5.6], the restriction of the ambient polarization is of type $(3,3)$. Moreover, the surface $A$ is the singular locus of $D_{Y_{6}^{S p}}$, the Pfaffian cubic hypersurface. By Theorem 2.1, this hypersurface must be the Coble cubic $\mathcal{C}_{3}$. Of course all these loci depend on $\omega$, but we will omit this dependence in our notation.

The geometry of the pair $\left(A, \mathcal{C}_{3}\right)$ was described in more details in [GS15]. For example, it can be proved that $A$ parametrizes the family of $\mathbf{P}^{4}$ 's contained in $\mathcal{C}_{3}$. Moreover, each such $\mathbf{P}^{4}$ cuts $A$ along a theta-divisor [GS15, Theorem $3.6]$.

By varying $\omega$, one gets a locally complete family of $(3,3)$-polarized abelian surfaces [GSW13].

On the dual space $\mathbf{P}\left(V_{9}\right)$, notice that $\omega \in \wedge^{3} V_{9} \cong \mathrm{H}^{0}\left(\mathbf{P}\left(V_{9}\right), \wedge^{3} \mathcal{Q}\right)$, where $\mathcal{Q}$ denotes the tautological quotient bundle (of rank eight). The fiber of $\wedge^{3} \mathcal{Q}$ is isomorphic to $\wedge^{3} \mathbb{C}^{8}$. Just as we did on $\mathbf{P}\left(V_{9}^{\vee}\right)$ when we defined the Pfaffian loci, we can define subvarieties of $\mathbf{P}\left(V_{9}\right)$ as loci where the trivector that we obtain on $\mathcal{Q}$ has some special behavior, in the sense that it belongs to some proper $G L_{8}$-orbit (or rather, orbit closure) in $\wedge^{3} \mathbb{C}^{8}$. This is precisely the idea behind the notion of orbital degeneracy loci introduced in [BFMT17, BFMT18]. The next section will be devoted to the study of the relevant orbits in $\wedge^{3} \mathbb{C}^{8}$; in the last sections, we will provide geometric interpretations for the corresponding orbital degeneracy loci in $\mathbf{P}\left(V_{9}\right)$.

\section{The affine model: trivectors in eight variables}

Our model will be the $G L_{8}$-representation $\wedge^{3} V_{8}$. This is a classical example of a representation with a finite number of orbits. The properties we will need in the following regarding this space can be found in [Gur64, KW]. 
We denote by $Y_{i}$ a codimension $i$ orbit closure inside $\wedge^{3} V_{8}$. As we want to construct some orbital degeneracy loci (see Section 4.1) inside $\mathbf{P}\left(V_{9}\right)$, whose dimension is eight, we will focus on the varieties $Y_{i}$ for $i \leq 8$. As it turns out, there is exactly one orbit closure of codimension $i$ for $i=1,3,4,6$ and two distinct orbit closures $Y_{8}$ and $Y_{8}^{\prime}$ of codimension eight. The inclusion diagram is given in Figure 1.

Figure 1: Inclusions of orbit closures up to codimension eight in $\wedge^{3} V_{8}$

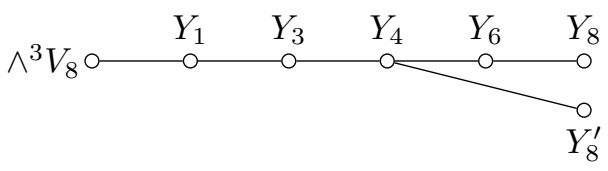

\subsection{Kempf collapsings}

In $[\mathrm{KW}]$, the geometry of these orbit closures has been studied with the help of birational Kempf collapsings. These are particular resolutions of singularities given by total spaces of homogeneous vector bundles on some auxiliary flag manifolds.

Let $F=G / P$ such a flag manifold, for $P$ a parabolic subgroup of an algebraic group $G$. Then a homogeneous bundle on $F$ is of the form $\mathcal{E}_{U}=G \times{ }_{P} U$ for some $P$-module $U$. If $U$ is a $P$-submodule of a $G$-module $V$, then $\mathcal{E}_{U}$ is a sub-vector bundle of $\mathcal{E}_{V}$, which is the trivial bundle on $F$ with fiber $V$. In particular, if we denote by $W$ the total space of $\mathcal{E}_{U}$, this construction induces a proper $G$-equivariant map $\pi_{U}: W \rightarrow V$, called a Kempf collapsing. When $V$ has only finitely many $G$-orbits (e.g., the $G L_{8}$-representation $\wedge^{3} V_{8}$ ), the image of $\pi_{U}$ must be some orbit closure $Y$. In many cases, $Y$ being given, we can always find a parabolic $P$ and a $P$-module $U$ such that the image of $\pi_{U}$ is $Y$.

Partially following [KW], in Table 2 we provide a few finite Kempf collapsings for the biggest orbit closures in $V=\wedge^{3} V_{8}$, together with their degrees. We denote by $F\left(r_{1}, \ldots, r_{i}, \ldots, V_{8}\right)$ the variety parametrizing flags of subspaces of $V_{8}$ of dimensions $r_{1}, \ldots, r_{i}$. On this flag manifold, we denote by $\mathcal{U}_{r_{i}}$ the tautological bundle of rank $r_{i}$.

Table 2: Some $d: 1$ Kempf collapsings for the orbit closures in $\wedge^{8} V_{8}$ of codimension up to 8 .

\begin{tabular}{cccr}
\hline$Y$ & $F$ & $\mathcal{E}_{U}$ & $d$ \\
\hline$Y_{1}$ & $\operatorname{Gr}\left(5, V_{8}\right)$ & $\wedge^{2} \mathcal{U}_{5} \wedge V_{8}$ & 1 \\
$Y_{3}$ & $F\left(1,4, V_{8}\right)$ & $\mathcal{U}_{1} \wedge\left(\wedge^{2} V_{8}\right)+\left(\wedge^{2} \mathcal{U}_{4}\right) \wedge V_{8}$ & 2 \\
$Y_{4}$ & $F\left(2,5, V_{8}\right)$ & $\wedge^{3} \mathcal{U}_{5}+\mathcal{U}_{2} \wedge \mathcal{U}_{5} \wedge V_{8}$ & 1 \\
$Y_{4}$ & $F\left(4,6, V_{8}\right)$ & $\wedge^{2} \mathcal{U}_{4} \wedge V_{8}+\wedge^{3} \mathcal{U}_{6}$ & 3 \\
$Y_{4}$ & $F\left(2,4,5,6, V_{8}\right)$ & $\mathcal{U}_{2} \wedge\left(\mathcal{U}_{4} \wedge V_{8}+\mathcal{U}_{5} \wedge \mathcal{U}_{6}\right)+\wedge^{3} \mathcal{U}_{5}$ & 3 \\
$Y_{6}$ & $F\left(1,3,4,6,7, V_{8}\right)$ & $\mathcal{U}_{1} \wedge\left(\mathcal{U}_{4} \wedge V_{8}+\wedge^{2} \mathcal{U}_{6}\right)+\mathcal{U}_{3} \wedge\left(\mathcal{U}_{3} \wedge \mathcal{U}_{7}+\mathcal{U}_{4} \wedge \mathcal{U}_{6}\right)$ & 1 \\
$Y_{8}$ & $F\left(2,5,7, V_{8}\right)$ & $\mathcal{U}_{2} \wedge\left(\mathcal{U}_{2} \wedge V_{8}+\mathcal{U}_{5} \wedge \mathcal{U}_{7}\right)+\wedge^{3} \mathcal{U}_{5}$ & 1 \\
$Y_{8}^{\prime}$ & $\operatorname{Gr}\left(2, V_{8}\right)$ & $\mathcal{U}_{2} \wedge\left(\wedge^{2} V_{8}\right)$ & 1 \\
\hline
\end{tabular}


Remark 3.1. The Kempf collapsing corresponding to $Y_{3}$ is finite by a dimension count. Its degree is at least 2: indeed, by [Gur64], a general element of $Y_{3}$ is $y_{3}=v_{123}+v_{456}+v_{147}+v_{268}+v_{358}$ and at least the two flags $\left\langle v_{1}\right\rangle \subset\left\langle v_{1}, v_{5}, v_{6}, v_{8}\right\rangle$ and $\left\langle v_{4}\right\rangle \subset\left\langle v_{2}, v_{3}, v_{4}, v_{8}\right\rangle$ are in the preimage of $y_{3}$ in the total space of $\mathcal{U}_{1} \wedge$ $\left(\wedge^{2} V_{8}\right)+\left(\wedge^{2} \mathcal{U}_{4}\right) \wedge V_{8}$ over $F\left(1,4, V_{8}\right)$. A direct computation in the proof of Proposition 5.4 will show that it is exactly 2 .

In Proposition 3.2 we will show that the second Kempf collapsing for $Y_{4}$ appearing in Table 2 is indeed of degree 3 . The third one will appear as the fiber product of the first two at the end of Section 3.2.

Notation. For a flag $V_{a} \subset V_{b} \subset V_{c}$, we will write $V_{a b c}:=V_{a} \wedge V_{b} \wedge V_{c}$ for short. For instance, for $y \in \wedge^{3} V_{8}$, it turns out that $y \in Y_{3}$ if and only if $y \in V_{188}+V_{448}$ for some flag $V_{1} \subset V_{4} \subset V_{8}$ (see Remark 3.1), meaning that $y$ can be decomposed into a sum of elements of $V_{188}$ and $V_{448}$.

A birational Kempf collapsing should be interpreted in the following way: for each point $\omega$ of the open orbit in $Y$, there exists a unique flag $V_{\bullet} \in F$ such that $\omega$ belongs to the fiber of $\mathcal{E}_{U}$ over $V_{\bullet}$. In some sense this yields a normal form for $\omega$. It is mainly from this perspective that in the sequel we will make use of Kempf collapsings.

Let us mention some of the properties of the orbit closures $Y_{i} \subset \wedge^{8} V_{8}$ that will be useful to us in the sequel.

- The orbit closures $Y_{1}, Y_{3}, Y_{4}, Y_{8}$ are normal, Cohen-Macaulay, and have rational singularities; only $Y_{1}$ and $Y_{4}$ are Gorenstein. $Y_{6}$ and $Y_{8}^{\prime}$ are neither normal nor Cohen-Macaulay.

- Any other orbit of codimension higher than eight is contained in $Y_{8} \cup Y_{8}^{\prime}$.

- $Y_{1}$ is the hypersurface defined by the hyperdeterminant, the unique $S L_{8}$ invariant polynomial of degree 16 over $\wedge^{3} V_{8}$.

- $Y_{3}$ is the singular locus of $Y_{1}$.

- The structure sheaf of $Y_{4}$ admits the following self-dual resolution:

$$
\begin{gathered}
0 \rightarrow \operatorname{det}\left(V_{8}^{\vee}\right)^{9} \rightarrow \operatorname{Sym}^{2} V_{8}^{\vee} \otimes \operatorname{det}\left(V_{8}^{\vee}\right)^{5} \rightarrow \wedge^{4} V_{8}^{\vee} \otimes \operatorname{det}\left(V_{8}^{\vee}\right)^{4} \rightarrow \\
\rightarrow \operatorname{Sym}^{2} V_{8} \otimes \operatorname{det}\left(V_{8}^{\vee}\right)^{4} \rightarrow \mathcal{O}_{\wedge^{3} V_{8}} \rightarrow \mathcal{O}_{Y_{4}} \rightarrow 0 .
\end{gathered}
$$

\subsection{Two triple covers}

A rather delicate but very interesting point is that there are a priori more Kempf collapsings than orbit closures. It can happen that some orbit closures have several resolutions of singularities by different Kempf collapsings. It can also happen that a Kempf collapsing is not birational onto its image, either because the dimension drops or, more scarcely, because it has positive degree. Although the latter phenomenon cannot happen for the Kempf collapsing of a completely reducible homogeneous vector bundle ([Kem76, Proposition 2 (c)]), we already met an instance of it in Remark 3.1. The next example will be essential in the sequel: 
Proposition 3.2. On the flag manifold $F_{2}:=F\left(4,6, V_{8}\right)$, consider the homogeneous vector bundle $\mathcal{E}_{2}=\wedge^{2} \mathcal{U}_{4} \wedge V_{8}+\wedge^{3} \mathcal{U}_{6}$. Then the Kempf collapsing $\pi_{2}$ of its total bundle $W_{2}$ is a generically $3: 1$ cover of $Y_{4}$.

Proof. Recall from Table 2 that $Y_{4}$ admits a resolution of singularities $\pi_{1}$ : $W_{1} \rightarrow Y_{4}$, where $W_{1}$ is the total space of the vector bundle $\wedge^{3} \mathcal{U}_{5}+\mathcal{U}_{2} \wedge \mathcal{U}_{5} \wedge V_{8}$ on $F_{1}:=F\left(2,5, V_{8}\right)$. Being equivariant, it must be an isomorphism on the open orbit $\mathcal{O}_{4}$. We will show that

i. if $y \in \pi_{2}\left(F_{2}\right)$ (or, equivalently, if there exist $U_{4} \subset U_{6} \subset V_{8}$ such that $\left.y \in \wedge{ }^{2} U_{4} \wedge V_{8}+\wedge{ }^{3} U_{6}\right)$, then there exists a flag $V_{2} \subset V_{5}$ with $V_{2} \subset U_{4} \subset$ $V_{5} \subset U_{6}$ such that $y \in V_{555}+V_{258}$. In particular, $\pi_{2}\left(F_{2}\right) \subset Y_{4}$;

ii. if $y \in \mathcal{O}_{4}$, then there exist exactly three flags $U_{4} \subset U_{6}$ such that $y \in$ $U_{448}+U_{666}$. In particular, $\mathcal{O}_{4} \subset \pi_{2}\left(F_{2}\right)$.

These two claims will imply that $\pi_{2}\left(F_{2}\right)=Y_{4}$ and that $\pi_{2}$ is a $3: 1$ over $\mathcal{O}_{4}$.

To prove i., we fix $U_{4} \subset U_{6}$ and we consider the space of parameters for $V_{2} \subset$ $V_{5}$ with $V_{2} \subset U_{4} \subset V_{5} \subset U_{6}$, i.e., $\operatorname{Gr}\left(2, U_{4}\right) \times \mathbf{P}\left(U_{6} / U_{4}\right)$. Over this space, the point $y$ can be regarded as a section of the trivial vector bundle $\wedge^{2} U_{4} \wedge V_{8}+\wedge^{3} U_{6}$; on a point $V_{2} \subset V_{5}, y \in V_{2} \wedge U_{4} \wedge U_{8}+\wedge^{3} U_{6}$ if and only if the induced section $\bar{y}$ on the quotient bundle $V_{8} / V_{6} \otimes \wedge^{2}\left(U_{4} / V_{2}\right)$ vanishes. On the points where $\bar{y}$ vanishes, $y$ induces a section of $V_{258}+\wedge^{3} U_{6}$; it will belong to $V_{258}+\wedge^{3} V_{5}$ if and only the induced section on $\wedge^{3} U_{6} /\left(V_{258}+\wedge^{3} U_{5}\right)=\left(U_{6} / V_{5}\right) \otimes \wedge^{2}\left(V_{5} / U_{2}\right)$ vanishes. In other words, the points in $\operatorname{Gr}\left(2, U_{4}\right) \times \mathbf{P}\left(U_{6} / U_{4}\right)$ in the zero locus of the section induced by $y$ of the vector bundle

$$
E=\wedge^{2}\left(U_{4} / V_{2}\right)^{\oplus 2} \oplus \wedge^{2}\left(U_{5} / V_{2}\right)
$$

will give the flags we are looking for. A straightforward computation shows that $c_{5}(E)=1$, so there exists at least one solution to our problem, as claimed.

To prove ii., we start with a point $y \in \mathcal{O}_{4}$, for which there exists a unique flag $V_{2} \subset V_{5}$ inside $F_{1}$ such that $y \in V_{258}+V_{555}$. By i., any flag $U_{4} \subset U_{6}$ such that $y \in U_{448}+U_{666}$ has to satisfy $V_{2} \subset U_{4} \subset V_{5} \subset U_{6}$. This can be reformulated as the condition that contracting $y$ with any element $\eta \in U_{6}^{\perp}$, we get an element of $\wedge^{2} U_{4}$. Note that since $y \in V_{2} \wedge V_{5} \wedge V_{8}+\wedge^{3} V_{5}$, such a contraction will belong to $V_{2} \wedge V_{5}$. So it belongs to $\wedge^{2} U_{4}$ if and only if its image in $V_{2} \otimes\left(V_{5} / V_{2}\right)$ is contained in $V_{2} \otimes\left(U_{4} / V_{2}\right)$. Dualizing, we need that the induced morphism from $U_{6}^{\perp} \otimes V_{2}^{\vee}$ to $V_{5} / V_{2}$ has rank two, which occurs in codimension two, that is, for a finite number of spaces $U_{6}$. Then $U_{4} / V_{2}$ (hence $U_{4}$ ) is determined as the image of the previous morphism. We conclude that the number of solutions to our problem can be computed on $\mathbf{P}\left(V_{8} / V_{5}\right)$, by the Thom-Porteous formula, as the class

$$
c_{2}\left(U_{6}^{\perp} \otimes V_{2}^{\vee}-V_{5} / V_{2}\right)=c_{2}\left(U_{6}^{\perp} \otimes V_{2}^{\vee}\right)=3 .
$$

Being equivariant, $\pi_{2}$ must be finite and étale over the open orbit $\mathcal{O}_{4}$ in $Y_{4}$, hence there exist exactly three flags over any point in the orbit.

Example 3.3. Let us denote again by $W_{1}$ the (total space of the) vector bundle $\wedge^{3} \mathcal{U}_{5}+\mathcal{U}_{2} \wedge \mathcal{U}_{5} \wedge \mathcal{U}_{8}$ over $F\left(2,5, V_{8}\right)$, which yields a Kempf collapsing resolving $Y_{4}$. Consider the point $y_{4}=v_{456}+v_{147}+v_{257}+v_{268}+v_{358}+v_{367}$ of $\mathcal{O}_{4}$, see [Gur64]. Its unique preimage in $W_{1}$ is given by the flag

$$
V_{2}=\left\langle v_{7}, v_{8}\right\rangle \quad \subset \quad V_{5}=\left\langle v_{4}, v_{5}, v_{6}, v_{7}, v_{8}\right\rangle .
$$


Its three preimages in $W_{2}$ are given by

$$
\begin{aligned}
U_{4}=\left\langle v_{5}, v_{6}, v_{7}, v_{8}\right\rangle & \subset U_{6}=\left\langle v_{1}, v_{4}, v_{5}, v_{6}, v_{7}, v_{8}\right\rangle, \\
U_{4}=\left\langle v_{4}, v_{5}-v_{6}, v_{7}, v_{8}\right\rangle & \subset U_{6}=\left\langle v_{2}+v_{3}, v_{4}, v_{5}, v_{6}, v_{7}, v_{8}\right\rangle, \\
U_{4}=\left\langle v_{4}, v_{5}+v_{6}, v_{7}, v_{8}\right\rangle & \subset U_{6}=\left\langle v_{2}-v_{3}, v_{4}, v_{5}, v_{6}, v_{7}, v_{8}\right\rangle .
\end{aligned}
$$

Let $y$ be a point in $Y_{4}$, and consider a flag $V_{2} \subset V_{4} \subset V_{5} \subset V_{6}$ defining points of $W_{1}$ and $W_{2}$ over $y$. This means that $y$ belongs to the intersection of $\wedge{ }^{3} V_{5}+V_{2} \wedge V_{5} \wedge V_{8}$ with $\wedge{ }^{3} V_{6}+\wedge{ }^{2} V_{4} \wedge V_{8}$, that is, to $V_{248}+V_{256}+V_{555}$. This suggests to consider, on the flag manifold $F\left(2,4,5,6, V_{8}\right)$, the vector bundle $\mathcal{U}_{248}+\mathcal{U}_{256}+\mathcal{U}_{555}$, and to denote its total space by $W_{12}$. Note that the latter bundle has rank 27 on a 25 -dimensional flag manifold, so that $W_{12}$ has dimension 52 , as expected.

We get the following diagram of morphisms:

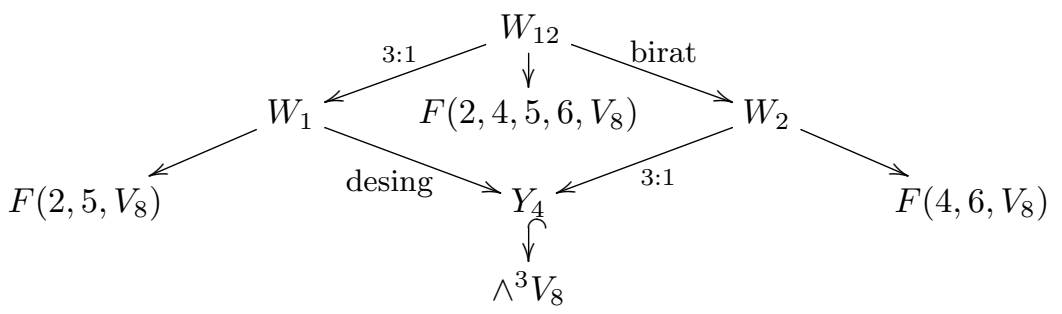

where $W_{12} \rightarrow W_{1}$ is generically $3: 1$.

\section{The degeneracy loci}

\subsection{Orbital degeneracy loci: generalities}

Let us briefly recall how orbital degeneracy loci are constructed [BFMT17, BFMT18]. One starts with a model, that we will choose to be a representation $V$ of some algebraic group $G$. Inside $V$, we consider a closed $G$-stable subvariety $Y$, usually the closure of a $G$-orbit. For any $G$-principal bundle $\mathcal{E}$ over a variety $X$, we can consider its associated vector bundle $\mathcal{E}_{V}$ on $X$. By construction, each fiber of this bundle gets identified with $V$, not canonically, but the ambiguity only comes from the action of $G$. This allows us to define, for any global section $s$ of $\mathcal{E}_{V}$, the orbital degeneracy locus

$$
D_{Y}(s)=\left\{x \in X \text { s.t. } s(x) \in Y \subset V \simeq \mathcal{E}_{V, x}\right\} .
$$

In the algebraic context, there is a natural scheme structure induced on $D_{Y}(s)$ that we will not consider. In the usual situation where $s$ is general in a finitedimensional space of global sections that generates $\mathcal{E}_{V}$ everywhere, the orbital degeneracy loci are well-behaved, in the sense that their properties faithfully reflect the properties of $Y$. In particular, $\operatorname{Sing}\left(D_{Y}(s)\right)=D_{\operatorname{Sing}(Y)}(s)$ and

$$
\operatorname{codim}_{X} D_{Y}(s)=\operatorname{codim}_{V} Y, \quad \operatorname{codim}_{D_{Y}(s)} \operatorname{Sing}\left(D_{Y}(s)\right)=\operatorname{codim}_{Y} \operatorname{Sing}(Y) .
$$

A remarkable feature of an orbital degeneracy locus $D_{Y}(s)$ associated to a subvariety $Y$ admitting a Kempf collapsing is that it is easy to relativize such a collapsing to get a surjective map $\mathscr{Z} \rightarrow D_{Y}(s)$. $\mathscr{Z}$ turns out to be the zero locus of an induced section of a vector bundle on a manifold, both determined by the collapsing. Moreover, if the Kempf collapsing is finite, such a map will be finite as well. We refer to [BFMT17, BFMT18] for more details. 


\subsection{Loci associated to an alternate trivector}

An example of orbital degeneracy locus is the abelian surface $A=D_{Y_{4}^{S p}}$ constructed in Section 2.3. Recall that $A$ is in fact only a torsor over an abelian surface, as it has no fixed origin; in order to simplify the terminology, from now on we will abusively call it the abelian surface $A$. Here the model $V$ is the space of alternating bivectors $\wedge^{2} V_{8}$, on which the group $G L_{8}$ acts. The stable closed subvarieties are the loci $Y_{r}^{S p}$ where the rank is bounded above by $r$. The trivector $\omega \in \wedge^{3} V_{9}$ defines a section of $\wedge^{2} H(1)$ over $\mathbf{P}\left(V_{9}^{\vee}\right)$, where $H$ denotes the tautological bundle on $\mathbf{P}\left(V_{9}^{\vee}\right)$, and the corresponding orbital degeneracy loci are the Pfaffian loci $D_{Y_{r}^{s p}} \subset \mathbf{P}\left(V_{9}^{\vee}\right)$.

On the dual projective space $\mathbf{P}\left(V_{9}\right)$, the trivector $\omega \in \wedge^{3} V_{9}$ can be seen as a section of $\wedge^{3} \mathcal{Q}, \mathcal{Q}$ being the tautological quotient bundle on $\mathbf{P}\left(V_{9}\right)$. For $Y_{i}$ the orbit closures inside $\wedge^{3} V_{8}$ introduced in Section 3 , the associated degeneracy loci are

$$
D_{Y_{i}}:=\left\{\left[V_{1}\right] \in \mathbf{P}\left(V_{9}\right) \text { s.t. } \omega\left(\bmod V_{1}\right) \in Y_{i} \subset \wedge^{3}\left(V_{9} / V_{1}\right)\right\}
$$

where we omit, for simplicity, the dependence on $\omega$. We will often write $\omega / V_{1}$ instead of $\omega\left(\bmod V_{1}\right)$ where no confusion can arise.

For a generic $\omega, D_{Y_{i}}$ has codimension $i$ inside $\mathbf{P}\left(V_{9}\right)$ and $\operatorname{Sing}\left(D_{Y_{i}}\right)=$ $D_{\operatorname{Sing}\left(Y_{i}\right)}$ (see Figure 3 for the inclusion graph). For example, $D_{Y_{1}}$ is a sextic hypersurface inside $\mathbf{P}\left(V_{9}\right)$, singular along $D_{Y_{3}}$, which is five-dimensional.

Figure 3: Inclusions of degeneraci locy inside $\mathbf{P}\left(V_{9}\right)$

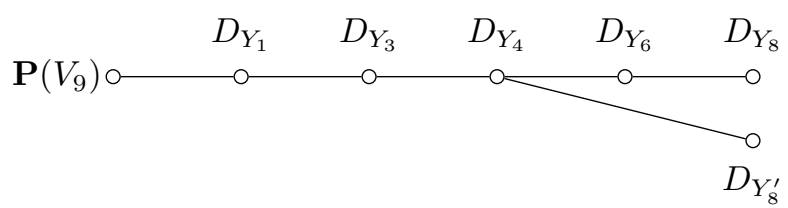

Proposition 4.1. $D_{Y_{1}}$ is the Coble sextic $\mathcal{C}_{6}$.

Proof. Let us prove that $D_{Y_{1}}$ is the dual hypersurface to the Coble cubic $\mathcal{C}_{3}=$ $D_{Y_{6}^{S p}}$. The conclusion will then follow from Theorem 2.2 .

A general point of the cubic hypersurface $D_{Y_{6}^{S p}}$ is a hyperplane $H$ such that we can decompose $\omega=e \wedge \theta+\sigma$ with $e \notin H, \sigma \in \wedge^{3} H$, and $\theta \in \wedge^{2} H$ is degenerate, i.e. $\theta^{4}:=\theta \wedge \theta \wedge \theta \wedge \theta=0$. At a smooth point of this hypersurface, $\theta$ has rank six. Let us analyze how $H$ can be deformed inside $D_{Y_{6}^{S p}}$.

We choose a basis $e_{1}, \ldots, e_{9}$ of $V_{9}$ such that $H$ is generated by $e_{1}, \ldots, e_{8}$, and $e_{9}=e$. On a neighborhood of $H$ in $\mathbf{P}\left(V_{9}^{\vee}\right)$, a hyperplane $H_{z}$ has a basis $f_{1}=e_{1}+z_{1} e_{9}, \ldots, f_{8}=e_{8}+z_{8} e_{9}$. In the basis $f_{1}, \ldots, f_{8}, e_{9}$ of $V_{9}$, our $\omega$ decomposes as $\left.e_{9} \wedge(\theta+z\lrcorner \sigma\right)(f)+\sigma(f)$, where $z=z_{1} e_{1}^{*}+\cdots+z_{8} e_{8}^{*}$ and $\theta(f), \sigma(f)$ are obtained by expressing $\theta, \sigma$ in the basis $e_{1}, \ldots, e_{8}$, and replacing formally each $e_{i}$ by $f_{i}$. So $H_{z}$ remains inside $D_{Y_{6}^{S p}}$ if and only if $\left.(\theta+z\lrcorner \sigma\right)^{4}=0$. In particular, the tangent hyperplane to $D_{Y_{6}^{S p}}$ at $H$ is given by the condition that $\left.\theta^{3} \wedge(z\lrcorner \sigma\right)=0$. 
Suppose our basis has been chosen so that $\theta=e_{12}+e_{34}+e_{56}$, and decompose further our tensor as

$$
\omega=e_{9} \wedge\left(e_{12}+e_{34}+e_{56}\right)+e_{7} \wedge \sigma_{7}+e_{8} \wedge \sigma_{8}+e_{7} \wedge e_{8} \wedge \sigma_{78}
$$

where $\sigma_{7}, \sigma_{8}, \sigma_{78}$ only involve $V_{6}:=\left\langle e_{1}, \ldots, e_{6}\right\rangle$. The condition $\left.\theta^{3} \wedge(z\lrcorner \sigma\right)=0$ simply becomes $z\lrcorner \sigma_{78}=0$, or equivalently, that $\sigma_{78}$ belongs to $H_{z}$. In other words, the tangent hyperplane to $D_{Y_{6}^{S p}}$ at $[H]$ is the orthogonal hyperplane to the vector $\sigma_{78}$.

Finally, we claim that $\left[\sigma_{78}\right]$ belongs to $D_{Y_{1}}$. Indeed, when we mod out by $\sigma_{78}$, we get

$$
\bar{\omega}=e_{9} \wedge\left(e_{12}+e_{34}+e_{56}\right)+e_{7} \wedge \sigma_{7}+e_{8} \wedge \sigma_{8},
$$

now in $\wedge^{3}\left(V_{9} /\left\langle\sigma_{78}\right\rangle\right)$ (with some abuse of notation), and the factors of $e_{9}, e_{7}, e_{8}$ now live in $\wedge^{2}\left(V_{6} /\left\langle\sigma_{78}\right\rangle\right)$. If $\bar{V}_{5}:=V_{6} /\left\langle\sigma_{78}\right\rangle$ and $\bar{V}_{8}:=V_{9} /\left\langle\sigma_{78}\right\rangle$, we conclude that $\bar{\omega}$ belongs to $\wedge^{2} \bar{V}_{5} \wedge \bar{V}_{8}$. But this is precisely the condition that defines $D_{Y_{1}}$. We have thus proved that the dual of $\mathcal{C}_{3}=D_{Y_{6}^{S p}}$ is contained in $D_{Y_{1}}$.

Conversely, let us consider a general point $\left[V_{1}\right]$ of $D_{Y_{1}}$, generated by $e_{1}$. From the Kempf collapsing resolving $Y_{1}$, we see that this means that there must exist a unique $V_{6}$, with $V_{1} \subset V_{6} \subset V_{9}$, such that $\omega$ belongs to $V_{199}+V_{669}$. This is equivalent to the fact that the contraction by $\omega$ sends $\wedge^{2} V_{6}^{\perp}$ to $V_{1}$.

Let us consider a basis $e_{1}, \ldots, e_{6}$ of $V_{6}$. Note that $\left(V_{199}+V_{669}\right) / V_{669}$ is isomorphic to $V_{1} \otimes \wedge^{2}\left(V_{9} / V_{6}\right)$. Since $V_{9} / V_{6}$ is three-dimensional, every bivector in $\wedge^{2}\left(V_{9} / V_{6}\right)$ is decomposable. This allows us to complete our basis of $V_{6}$ in a basis of $V_{9}$ with three vectors $e_{7}, e_{8}, e_{9}$ in $V_{9}$ such that

$$
\omega=e_{178}+\phi_{7} \wedge e_{7}+\phi_{8} \wedge e_{8}+\phi_{9} \wedge e_{9}+\psi,
$$

where $\phi_{7}, \phi_{8}, \phi_{9}$ belong to $\wedge^{2} V_{6}$ and $\psi$ to to $\wedge^{3} V_{6}$. We claim that the tangent space to $D_{Y_{1}}$ at $\left[V_{1}\right]$ is the hyperplane defined by the linear form $e_{9}^{*}$ from the dual basis. Indeed, we describe points $\left[U_{1}\right]$ in $D_{Y_{1}}$ locally around $\left[V_{1}\right]$ by moving $V_{6}$ to spaces $U_{6}$ such that the contraction by $\omega$ from $\wedge^{2} U_{6}^{\perp}$ to $V_{9}$ keeps rank one, and defining $U_{1}$ as the image. Locally around $V_{6}$, such a space $U_{6}$ is defined by linear forms $f_{i}^{*}=e_{i}^{*}+t_{i}$, for $i=7,8,9$, where $t_{i}$ is a linear combination of $e_{1}^{*}, \ldots, e_{6}^{*}$. Clearly the contraction $\omega\left(f_{7}^{*}, f_{8}^{*}, \cdot\right)$ is a non-zero vector, which must therefore generate $U_{1}$, and its coefficient on $e_{9}$ is $\phi_{9}\left(t_{7}, t_{8}\right)$, which has order two. Modding out order two deformations, $U_{1}$ is thus contained in $\left\langle e_{1}, \ldots, e_{8}\right\rangle$, which implies the claim.

In order to conclude the proof, we just need to check that this tangent hyperplane belongs to $\mathcal{C}_{3}=D_{Y_{6}^{S p}}$, or equivalently, that the contraction $\omega\left(e_{9}^{*}, \cdot, \cdot\right)$ has rank at most six. But that is clear, since this contraction is $\phi_{9}$, an element of $\wedge^{2} V_{6}$.

Corollary 4.2. $D_{Y_{3}}$ is the singular locus of the sextic $\mathcal{C}_{6}$.

Proposition 4.3. There exists a natural birational map $D_{Y_{6}}-\rightarrow A$.

Proof. Let $\left[e_{0}\right]$ be a point in $D_{Y_{6}}$. By definition, this means that we can decompose $\omega$ with respect to a decomposition $V_{9}=\mathbb{C} e_{0} \oplus V_{8}$ as $\omega=e_{0} \wedge \alpha+\eta$, where $\eta$ belongs to $Y_{6} \subset \wedge^{3} V_{8}$. By Table 2 , for $\left[e_{0}\right]$ outside $D_{Y_{8}}$, this implies that there exists a unique flag $V_{1} \subset V_{4} \subset V_{7} \subset V_{8}$ such that $\eta$ belongs to $V_{148}+\wedge^{3} V_{7}$. Let 
$W_{8}=\mathbb{C} e_{0} \oplus V_{7}$. Let us also choose a generator $e_{1}$ of $V_{1}$, and some $e_{8} \notin W_{8}$. We can then write $\omega$ as

$$
\omega=e_{0} \wedge u \wedge e_{8}+e_{1} \wedge v \wedge e_{8}+\xi
$$

for some $u \in V_{7}, v \in V_{4}, \xi \in \wedge^{3} W_{8}$. Since the two-form $e_{0} \wedge u+e_{1} \wedge v$ has rank at most four, this implies that $W_{8}$ is a point of $A$. We have thus a rational map sending $\left[e_{0}\right]$ to $V_{7}\left(\left[e_{0}\right]\right) \oplus \mathbb{C} e_{0}$.

Conversely, we claim that for a general $P \in A$, there exists a unique line $l_{P} \subset P$ such that $\left(\omega / l_{P}\right) \in Y_{6} \subset \wedge^{3}\left(V_{9} / l_{P}\right)$ and $V_{7}\left(\left(\omega / l_{P}\right)\right)=P / l_{P}$. By hypothesis there exist $\sigma \in \wedge^{2} P$ of rank four and $\sigma^{\prime} \in \wedge^{3} P, v_{8} \notin P$ such that $\omega=v_{8} \wedge \sigma+\sigma^{\prime}$.

We want to show that there exists in general exactly one flag $V_{1} \subset V_{3} \subset$ $V_{4} \subset V_{6} \subset V_{7} \subset\left(V_{9} / l_{P}\right)$ such that $\omega / l_{P} \in V_{148}+V_{166}+V_{337}+V_{346}$. The space $V_{7}=P / l_{P}$ is determined by $l_{P}$. Denote by $W$ the four-dimensional space defined by $\sigma$; then, $l_{P} \subset W$ and therefore $\mathbf{P}(W)$ is the right parameter space for $l_{P}$. Indeed, the contraction of $\omega / l_{P}$ by any element of $\left(P / l_{P}\right)^{\perp}$ has to belong to $V_{1} \wedge V_{4}$, hence it has rank at most two, but this means that also the rank of $\sigma / l_{P}$ can be at most two. Moreover, the two-dimensional space $l_{P}^{\perp} / l_{P}$ defined by $\sigma / l_{P}$ must contain $V_{1}$ and be contained inside $V_{4}$. Therefore $V_{1}$ is parametrized by $\mathbf{P}\left(l_{P}^{\perp_{\sigma}} / l_{P}\right)$, while $V_{4} / l_{P}^{\perp \sigma} \subset V_{6} / l_{P}^{\perp_{\sigma}} \subset P / l_{P}^{\perp \sigma}$ is parametrized by $F\left(2,4, P / l_{P}^{\perp \sigma}\right)$ and $V_{3} / V_{1} \subset V_{4} / V_{1}$ is parametrized by $\operatorname{Gr}\left(2, V_{4} / V_{1}\right)$.

Inside this fourteen-dimensional parameter space, $\left(v_{8} \wedge \sigma\right) / l_{P}$ belongs by construction to $V_{148}$. If we interpret $\sigma^{\prime} / l_{P}$ as a section of the bundle $\wedge^{3}\left(P / l_{P}\right)$, the flags we are looking for are defined by the vanishing of the section that $\sigma^{\prime} / l_{P}$ induces on the quotient bundle $\wedge^{3} V_{7} /\left(V_{147}+V_{337}+V_{346}+V_{166}\right)$. To determine the number of such flags, we must compute the top Chern class of this quotient bundle. In order to do this, we first filter our bundle by homogeneous subbundles such that the associated graded bundle is completely reducible. Explicitely, the associated bundle we get is

$$
\begin{aligned}
\left(V_{7} / V_{6}\right) \otimes\left(V_{4} \otimes\left(V_{6} / V_{4}\right) \oplus \wedge^{2}\left(V_{6} / V_{4}\right) \oplus\left(V_{4} / V_{3}\right) \otimes\right. & \left.\left(V_{3} / V_{1}\right)\right) \oplus \\
& \oplus \wedge^{2}\left(V_{6} / V_{4}\right) \otimes\left(V_{4} / V_{1}\right) .
\end{aligned}
$$

A computation with [GS] shows that the top Chern class of the latter bundle is 1 , hence also of the original one, and the claim follows.

We have thus defined two rational maps $D_{Y_{6}} \rightarrow A$ and $A \rightarrow D_{Y_{6}}$, inverse one to the other. This implies the claim.

We will now focus on the four-dimensional orbital degeneracy locus $D_{Y_{4}} \subset$ $\mathbf{P}\left(V_{9}\right)$. A priori, it contains $D_{Y_{6}}$ (dimension two), $D_{Y_{8}}$ and $D_{Y_{8}^{\prime}}$ (dimension 0 ). Following [BFMT17, BFMT18], we can relativize the three Kempf collapsings of Table 2 and Section 3.2. We will denote by $Z$ the desingularization of $D_{Y_{4}}$ which is a zero locus inside the flag bundle $F(2,5, \mathcal{Q}) \cong F\left(1,3,6, V_{9}\right)$ and which relativizes the first Kempf collapsing; more precisely, it is the zero locus of a section of the vector bundle

$$
\wedge^{3} V_{9} /\left(\mathcal{U}_{199}+\mathcal{U}_{369}+\mathcal{U}_{666}\right)
$$

induced by $\omega$. Similarly, the relativization of the second one yields a generically $3: 1$ morphism to $D_{Y_{4}}$ from a variety $T$ which is a zero locus of a section of the 
vector bundle

$$
\wedge{ }^{3} V_{9} /\left(\mathcal{U}_{199}+\mathcal{U}_{559}+\mathcal{U}_{777}\right)
$$

inside $F(4,6, \mathcal{Q}) \cong F\left(1,5,7, V_{9}\right)$. Finally, we have also an associated zero locus $X$ of a section of the vector bundle

$$
\wedge^{3} V_{9} /\left(\mathcal{U}_{199}+\mathcal{U}_{359}+\mathcal{U}_{367}+\mathcal{U}_{666}\right)
$$

inside the flag bundle $F(2,4,5,6, \mathcal{Q}) \cong F\left(1,3,5,6,7, V_{9}\right)$ which relativizes the fiber product $W_{1} \times_{Y_{4}} W_{2}$, see (3.4). The situation is described in (4.4).

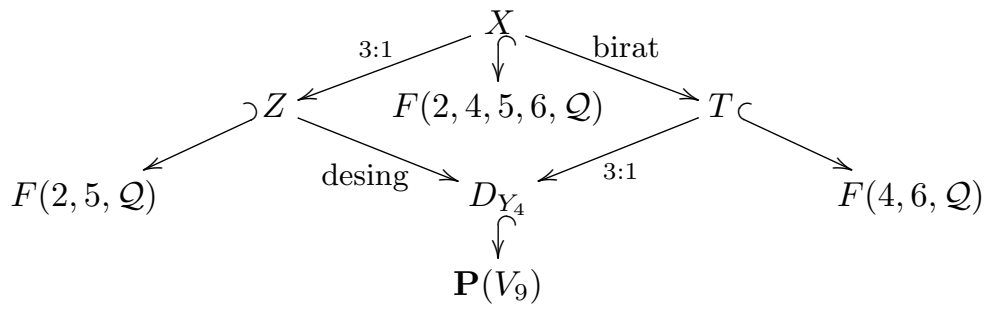

Remark 4.5. The varieties $Z, T, X$ depend on $\omega$. Once again, for the sake of lightness of notation, we will omit to write this dependence explicitly.

By using the description of the model $Y_{4}$ given in the previous section, the following facts can be checked.

Proposition 4.6. Let $\omega \in \wedge^{3} V_{9}$ be a generic trivector. Then:

1. $D_{Y_{8}}=\left\{p_{1}, \cdots, p_{81}\right\}$ consists in 81 reduced points, while $D_{Y_{8}^{\prime}}=\emptyset$.

2. The surface $D_{Y_{6}}$ is smooth outside $D_{Y_{8}}$.

3. $D_{Y_{4}}$ is a normal, linearly non-degenerate fourfold with $h^{i}\left(\mathcal{O}_{D_{Y_{4}}}\right)=1$ for $i=0,2,4$, and 0 otherwise.

4. $Z$ has trivial canonical bundle, $\chi\left(\mathcal{O}_{Z}\right)=3, \chi\left(\Omega_{Z}^{1}\right)=-6$ and $\chi\left(\Omega_{Z}^{2}\right)=90$.

5. $T$ also has trivial canonical bundle, but $\chi\left(\mathcal{O}_{T}\right)=\chi\left(\Omega_{T}^{1}\right)=0$.

Proof. Statements 1., 3., 4., 5. can be proved by using the desingularization of the loci given by the Kempf collapsings of the affine model, and by computing the corresponding Chern classes with [GS]. Statement 2. is a consequence of the fact that $\operatorname{Sing}\left(Y_{6}\right) \subset Y_{8} \cup Y_{8}^{\prime}$.

Corollary 4.7. $Z$ is a hyper-Kähler fourfold.

Proof. This follows from 4. and the Beauville-Bogomolov decomposition.

\section{The Kummer geometry of a trivector}

This section relates the loci we have constructed with the geometry of the abelian surface $A=D_{Y_{4}^{S p}}$. More precisely, in the four theorems of this section we will identify the varieties $D_{Y_{4}}, Z, T, X$ with four "classical" fourfolds that can be constructed from $A$. 


\subsection{The Kummer fourfold}

We will denote by $\operatorname{Kum}^{2}(A)$ the generalized Kummer hyper-Kähler fourfold associated to $A$. It is a subvariety of the Hilbert $\operatorname{scheme} \operatorname{Hilb}^{3}(A)$ of three points over $A$. Recall that there is a well-defined natural morphism $H C$ : $\operatorname{Hilb}^{3}(A) \rightarrow A^{(3)}$, called the Hilbert-Chow morphism. Composing with the sum map $A^{(3)} \rightarrow A$, we get a morphism $\operatorname{Hilb}^{3}(A) \rightarrow A$ whose fibers are all copies of $\operatorname{Kum}^{2}(A)$ (note that this ensures that the Kummer fourfold is not affected by the choice of the origin in our torsor $A$ ). The Kummer fourfold is a resolution of the singularities of the fiber $\Sigma \subset A^{(3)}$ of the sum morphism.

Theorem 5.1. $Z$ is isomorphic to the generalized $K u m m e r$ fourfold $\operatorname{Kum}^{2}(A)$.

Proof. We will construct a finite flat morphism $Z^{\prime} \rightarrow Z$ of degree three. It will induce a morphism from $Z$ to $\operatorname{Hilb}^{3}(A)$. Since $Z$ is hyper-Kähler, the composition $Z \rightarrow A$ with the sum map must be constant, otherwise we would get non-trivial one-forms on $Z$. Therefore our morphism factorizes through $\operatorname{Kum}^{2}(A)$. Finally, it will turn out to be birational. Since $Z$ is a minimal model, such a birational morphism must be an isomorphism.

Recall that $Z$ is embedded inside $F\left(1,3,6, V_{9}\right)$. Denote by $F_{Z}$ the restriction to $Z$ of the $\mathbf{P}^{2}$-bundle defined by the natural projection $F\left(1,3,6,8, V_{9}\right) \rightarrow$ $F\left(1,3,6, V_{9}\right)$. By definition, for any flag $V_{1} \subset V_{3} \subset V_{6} \subset V_{9}$ in $Z$, $\omega$ belongs to $V_{199}+V_{369}+V_{666}$. Modding out the latter bundle by $V_{199}+V_{339}+V_{666}$, we get the vector bundle $A_{2} \otimes B_{3} \otimes C_{3}$, where $A_{2}=V_{3} / V_{1}, B_{3}=V_{6} / V_{3}$ and $C_{3}=V_{9} / V_{6}$. Since $F_{Z}=\mathbf{P}_{Z}\left(V_{9} / V_{6}\right)^{\vee}$, the class $\bar{\omega}$ of $\omega$ in $A_{2} \otimes B_{3} \otimes C_{3}$ defines a morphism

$$
\hat{\omega}: A_{2}^{\vee}(-1) \longrightarrow B_{3}
$$

of vector bundles over $F_{Z}$. We define $Z^{\prime}$ as the first degeneracy locus $D_{1}(\hat{\omega})$. We will prove that $Z^{\prime} \rightarrow Z$ is finite of degree three, i.e., the fibers always have expected codimension. As they are determinantal, this implies that they are Cohen-Macaulay, hence the projection $Z^{\prime} \rightarrow Z$ is flat. It will further induce a morphism to $\operatorname{Hilb}^{3}(A)$ because the points in the fibers of $Z^{\prime} \rightarrow Z$ are defined by hyperplanes $V_{8}$ that must belong to $A$. Indeed, since $V_{8}$ contains $V_{6}$, when we mod out by $\wedge^{3} V_{8}$ the class of $\omega$ belongs to $\left(V_{18}+V_{36}\right) \otimes\left(V_{9} / V_{8}\right)$. When $V_{8}$ defines a point of $D_{1}(\hat{\omega})$, the term from $V_{36}$ has rank two modulo $V_{1}$, so that the image of $\omega$ in $V_{18}+V_{36}$ has rank at most four, which is exactly the condition for $V_{8}$ to belong to $A$.

There remains to check that the projection $Z^{\prime} \rightarrow Z$ is indeed finite of degree three. First note that on $\mathbf{P}\left(C_{3}^{\vee}\right)$, the morphism $\hat{\omega}: A_{2}^{\vee}(-1) \longrightarrow B_{3}$ is expected to drop rank in codimension two, hence on a finite scheme of length $c_{2}\left(B_{3}-\right.$ $\left.A_{2}^{\vee}(-1)\right)=s_{2}\left(A_{2}^{\vee}(-1)\right)=3$.

Let us prove that $D_{1}(\hat{\omega})$ cannot be positive dimensional. By what has been said before, $D_{1}(\hat{\omega})$ is contained in $A$. Moreover, it is defined as a subscheme of a projective plane by three quadrics, the $2 \times 2$-minors of the matrix $\hat{\omega}$. If it is not the whole plane, this immediately implies that $D_{1}(\hat{\omega})$ is contained in a conic. In any case, if $D_{1}(\hat{\omega})$ has positive dimension, it must contain a rational curve. Since $A$ does not contain any rational curve, we get a contradiction.

Finally, we have to prove that the morphism is birational; for this sake, we provide an explicit description of the image of the morphism on a general point and show that it is generically injective. Let $\left[e_{0}\right] \in D_{Y_{4}}$ be a general point. Let 
$V_{9}=\mathbb{C} e_{0} \oplus V_{8}$. Then $\omega=e_{0} \wedge \sigma+\sigma^{\prime}$, where $\sigma \in \wedge^{2} V_{8}$ and

$$
\sigma^{\prime}=v_{456}+v_{147}+v_{257}+v_{268}+v_{358}+v_{367}
$$

in a suitable basis of $V_{8}$. One readily checks that the hyperplanes $\left[v_{1}^{*}\right]^{\perp},\left[v_{2}^{*}+\right.$ $\left.v_{3}^{*}\right]^{\perp},\left[v_{2}^{*}-v_{3}^{*}\right]^{\perp}$ belong to $A$ and contain $V_{6}$, hence they must be the three points in $A$ which correspond to $\left[e_{0}\right]$ via the morphism. If we contract any two linear forms among $v_{1}^{*}, v_{2}^{*}+v_{3}^{*}, v_{2}^{*}-v_{3}^{*}$ with $\sigma^{\prime}$ we get zero, so the same contraction with $\omega$ yields a multiple of $e_{0}$ (non-zero, since $\sigma$ is general). This means that we can generically recover $\left[e_{0}\right]$ from its image in $\operatorname{Hilb}^{3}(A)$.

Theorem 5.2. $D_{Y_{4}}$ is projectively equivalent to $\Sigma$.

Proof. We want to compare the vertical projections in the diagram

$$
\begin{array}{ccc}
Z & \simeq & \operatorname{Kum}^{2}(A) \\
p \downarrow & & \downarrow^{q} \\
\mathbf{P}\left(V_{9}\right) \longleftarrow D_{Y_{4}} & & \Sigma \longrightarrow|3 \Theta|
\end{array}
$$

Recall that since $\operatorname{Kum}^{2}(A)$ has no holomorphic one-forms, its Picard group and its Néron-Severi group are the same. Moreover, by [Bea83, Proposition 8], the Néron-Severi group of the Kummer fourfold is

$$
N S\left(\operatorname{Kum}^{2}(A)\right)=\operatorname{Pic}\left(\operatorname{Kum}^{2}(A)\right)=\iota(N S(A)) \oplus \mathbb{Z} E,
$$

where the map $\iota$ is injective and $E$ is the exceptional divisor of the projection from $\operatorname{Kum}^{2}(A)$ to $\Sigma$. We will denote by $L^{[2]}$ the image of $L \in N S(A)$ by $\iota$. For $\omega$ generic the abelian surface $A$ is generic, so $N S(A)=\mathbb{Z} \Theta$. The projection $q$ is then defined by the full linear system $\left|\Theta^{[2]}\right|$.

We will show below that $p$ also contracts $E$, so that the pull-back $\mathcal{L}$ of the dual tautological line bundle on $\mathbf{P}\left(V_{9}\right)$ must be of the form $L^{[2]}$ for some $L \in \operatorname{Pic}(A)$, hence $\mathcal{L}=\ell \Theta^{[2]}$. By [BN01, Lemma 5.2],

$$
\chi\left(\operatorname{Kum}^{2}(A), \ell \Theta^{[2]}\right)=3\left(\begin{array}{c}
\ell+2 \\
2
\end{array}\right) .
$$

A computation with $[\mathrm{GS}]$ yields that $\chi(Z, \mathcal{L})=9$, hence $\ell=1$. Since $D_{Y_{4}}$ is linearly non-degenerate by Proposition $4.6, p$ must be defined by the full linear system $|\mathcal{L}| \simeq \mathbf{P}^{8}$. So $p$ and $q$ are the same maps, and the conclusion follows.

It remains to show that $p$ contracts $E$. Recall from the proof of Theorem 5.1 how we constructed an isomorphism from $Z$ to $\operatorname{Kum}^{2}(A)$ : for any $V_{1}$ in $D_{Y_{4}}$ and any flag $V_{1} \subset V_{3} \subset V_{6} \subset V_{9}$ such that $\omega$ belongs to $V_{199}+V_{369}+V_{666}$ (hence defining a point of $Z$ above $V_{1}$ ), we deduced an element $\bar{\omega}$ of $A_{2} \otimes B_{3} \otimes C_{3}$, where $A_{2}=V_{3} / V_{1}, B_{3}=V_{6} / V_{3}$ and $C_{3}=V_{9} / V_{6}$. Then we proved that the first degeneracy locus of the induced morphism $A_{2}^{\vee}(-1) \rightarrow B_{3}$ over $\mathbf{P}\left(C_{3}^{\vee}\right)$ defines a length three subscheme of $A$.

It will sufficient to show that the preimage $p^{-1}\left(D_{Y_{6}}\right)$ is a three-dimensional subscheme of $E$. Since $E$ is irreducible, the two are in facts equal and their image through $p$ is 2-dimensional. If $V_{1}$ is a general point of $D_{Y_{6}}$, then we can 
write $\omega / V_{1}$ as $v_{456}+v_{147}+v_{257}+v_{268}+v_{358}$ for a suitable choice of a basis of $V_{9} / V_{1}$. This determines the unique flag

$$
\begin{aligned}
\left\langle v_{8}\right\rangle \subset\left\langle v_{4}, v_{7}, v_{8}\right\rangle \subset\left\langle v_{4}, v_{5}, v_{7}, v_{8}\right\rangle & \subset\left\langle v_{2}, v_{4}, v_{5}, v_{6}, v_{7}, v_{8}\right\rangle \subset \\
& \subset\left\langle v_{1}, v_{2}, v_{4}, v_{5}, v_{6}, v_{7}, v_{8}\right\rangle \subset V_{9} / V_{1} .
\end{aligned}
$$

given by the desingularization of $Y_{6}$. As it turns out, any flag in the rational normal curve

$$
V_{3} / V_{1}=\left\langle v_{8}, \alpha v_{4}+\beta v_{7}\right\rangle \subset V_{6} / V_{1}=\left\langle v_{4}, v_{5}, v_{7}, v_{8}, \alpha v_{2}+\beta v_{6}\right\rangle
$$

is contained in $p^{-1}\left(V_{1}\right)$ since $\omega \in V_{199}+V_{369}+V_{666}$, hence the conclusion follows if we can prove that it is contained in $E$. On any such flag, (5.3) induces flags $A_{1} \subset A_{2}, B_{1} \subset B_{2} \subset B_{3}$ and $C_{1} \subset C_{2} \subset C_{3}$ such that

$$
\bar{\omega} \in A_{1} \otimes B_{2} \otimes C_{3}+A_{2} \otimes B_{1} \otimes C_{2}+\left(A_{1} \otimes B_{3}+A_{2} \otimes B_{2}\right) \otimes C_{1} .
$$

Then it is easy to see that the length three subscheme we get in $\mathbf{P}\left(C_{3}^{\vee}\right)$ has multiplicity two at the point defined by the hyperplane $C_{2}$ (note that this point is exactly the hyperplane $V_{8}$, uniquely defined by $V_{1} \in D_{Y_{6}}$ ). Since $E$ is precisely the locus of non-reduced schemes, we are done.

Let $P \in A$ be a hyperplane in $\mathbf{P}\left(V_{9}\right)$, and let $P_{4}$ be the four-dimensional subspace of $P$ such that $\omega(P, \cdot, \cdot) \in \wedge^{2} P_{4}$. Then:

Proposition 5.4. $D_{Y_{3}}$ is covered by a family of $\mathbf{P}^{3}$ parametrized by A. More precisely, for any point $P \in A$, we have that $\mathbf{P}\left(P_{4}\right) \subset D_{Y_{3}}$.

Proof. As $P \in A$ and by the definition of $P_{4}$, we know that

$$
\omega \in \wedge^{3} P+V_{9} \wedge\left(\wedge^{2} P_{4}\right) .
$$

In order to show that $\mathbf{P}\left(P_{4}\right) \subset D_{Y_{3}}$, we need to prove that for any $V_{1} \subset P_{4}$, there exist $U_{2} \subset U_{5} \subset V_{9}$ such that $V_{1} \subset U_{2}$ and $\omega \in U_{2} \wedge\left(\wedge^{2} V_{9}\right)+V_{9} \wedge\left(\wedge^{2} U_{5}\right)$. Indeed, if this happens, then $\omega$ modulo $V_{1}$ belongs to the total space of the vector bundle which gives a Kempf collapsing of $Y_{3}$ inside $V_{9} / V_{1}$ (see Table 2), and therefore $V_{1} \in D_{Y_{3}}$.

Let $V_{1} \subset P_{4}$. We construct $U_{5}$ as a subspace of $P$. Moreover, $\omega(P, \cdot, \cdot)$ is a two-form on $P_{4}$, and therefore we can consider the orthogonal $V_{1}^{\perp \omega} \subset P_{4}$ of $V_{1}$ inside $P_{4}$ with respect to this two-form. We construct $U_{2}$ as a subspace of $V_{1}^{\perp_{\omega}}$ containing $V_{1}$. The parameter space for $U_{2}$ is then $\mathbf{P}\left(V_{1}^{\perp_{\omega}} / V_{1}\right)$ and the parameter space for the pair $U_{2} \subset U_{5}$ is the Grassmannian bundle $\operatorname{Gr}\left(3, P / U_{2}\right)$ over $\mathbf{P}\left(V_{1}^{\perp \omega} / V_{1}\right)$, a variety of dimension ten.

Asking that $V_{1} \subset U_{2} \subset V_{1}^{\perp_{\omega}}$ implies that $\omega(P, \cdot, \cdot) \in U_{2} \wedge P_{4}$. Therefore we have that $\omega \in \wedge^{3} P+U_{2} \wedge\left(\wedge^{2} V_{9}\right)$. Let us consider the element $\tilde{\omega} \in \wedge^{3}\left(P / U_{2}\right)$ induced by $\omega$. Then $\omega \in U_{2} \wedge\left(\wedge^{2} V_{9}\right)+V_{9} \wedge\left(\wedge^{2} U_{5}\right)$ if and only if $\tilde{\omega} \in \wedge^{2}\left(U_{5} / U_{2}\right) \wedge$ $P / U_{2}$. Over our parameter space, $U_{5} / U_{2}$ is parametrized by the rank three tautological bundle $\mathcal{U}$ over $\operatorname{Gr}\left(3, P / U_{2}\right)$. As a consequence, requiring that $\tilde{\omega} \in$ $\wedge^{2} \mathcal{U} \wedge\left(P / U_{2}\right)$ is the same as asking that the induced section $\bar{\omega}$ of the vector bundle $F:=\wedge^{3}\left(P / U_{2}\right) /\left(\wedge^{2} \mathcal{U} \wedge\left(P / U_{2}\right)\right)$ vanishes. $F$ is a rank ten vector bundle over the ten-dimensional parameter space, and the zero locus of its general 
section $\bar{\omega}$ parametrizes the pairs $U_{2}, U_{5}$ such that $\omega \in U_{2} \wedge\left(\wedge^{2} V_{9}\right)+V_{9} \wedge\left(\wedge^{2} U_{5}\right)$. This zero locus consists in general of

$$
c_{10}(F)=2
$$

points, as a computation with [GS] shows; as it is nonempty, there exist $U_{2} \subset$ $U_{5}$ with the required properties, and $V_{1} \in D_{Y_{3}}$. This concludes the proof. (Note that the existence of different flags comes from the fact that the Kempf collapsing we used has degree $d>1$, see Table 2: the above computation actually shows that $d=2$, as stated in Remark 3.1.)

Remark 5.5. The singular locus of $S U_{C}(3)$ can be identified with $D_{Y_{3}}$. This singular locus is known to coincide with the set of strictly semistable rank three vector bundles with trivial determinant. A generic point of this set is a bundle $L \oplus E$, where $L$ is a line bundle (or a point of $A$ ) and $E$ is a rank two vector bundle such that $\operatorname{det}(E)=L^{-1}$. Therefore, having fixed $L$, this set contains

$$
\left\{E \text { of } \operatorname{rank} 2 \text { s.t. } \operatorname{det}(E)=L^{-1}\right\} \cong S U_{C}(2),
$$

which is a $\mathbf{P}^{3}$, see Section 2.2. This gives the family of $\mathbf{P}^{3}$ 's parametrized by $A$ covering $D_{Y_{3}}$ exhibited in Proposition 5.4. Moreover, each $\mathbf{P}^{3}$ contains a copy of $\operatorname{Kum}^{1}(A)$, the Kummer surface associated to $A$ : this gives the family of $\operatorname{Kum}^{1}(A)$ parametrized by $A$ covering $D_{Y_{4}} \cong \Sigma$.

Corollary 5.6. $D_{Y_{6}}$ is not normal, and $A$ is its normalization.

Proof. We know that $D_{Y_{6}}$ is the singular locus of $D_{Y_{4}}$, so by Theorem 5.2 it coincides with the set of triples of the form $(P, P,-2 P)$ in $\Sigma$. In particular there is a bijective morphism $A \rightarrow D_{Y_{6}}$, which implies that the normalization of $D_{Y_{6}}$ is isomorphic to $A$.

There just remains to prove that the singular locus of $\Sigma$ is not normal. Let us consider the following commutative diagram

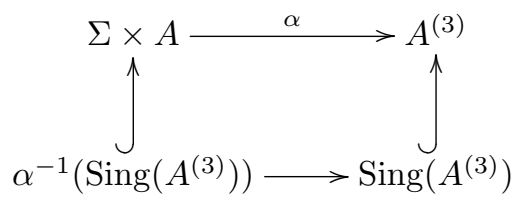

where $\alpha:([P, Q, R], t) \mapsto[P+t, Q+t, R+t]$. The preimage of a point is

$$
\alpha^{-1}\left(\left[P^{\prime}, Q^{\prime}, R^{\prime}\right]\right)=\left\{\left(\left[P^{\prime}-s, Q^{\prime}-s, R^{\prime}-s\right], s\right) \text { s.t. } 3 s=P^{\prime}+Q^{\prime}+R^{\prime}\right\} ;
$$

in particular, $\alpha$ is a $81: 1$ étale cover of $A^{(3)}$, and induces an étale cover of $\operatorname{Sing}\left(A^{(3)}\right)$ by the singular locus of $\Sigma \times A$. Consider the following diagram:

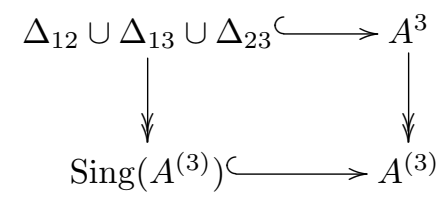

where, e.g., $\Delta_{12}=\left\{(P, P, Q) \in A^{3}\right\}$ is the first diagonal. The map on the left is generically $3: 1$, while the map on the right is generically $6: 1$. The restriction 
$\Delta_{12} \rightarrow \operatorname{Sing}\left(A^{(3)}\right)$ is a birational finite morphism, hence it is an isomorphism if $\operatorname{Sing}\left(A^{(3)}\right)$ is normal.

Locally, we have $A^{3} \cong\left(\mathbb{C}^{2}\right)^{3}$ and $\mathcal{O}_{A^{3}} \cong R:=\mathbb{C}\left[x_{1}, y_{1}, x_{2}, y_{2}, x_{3}, y_{3}\right]$. The above diagram induces the following commutative diagram

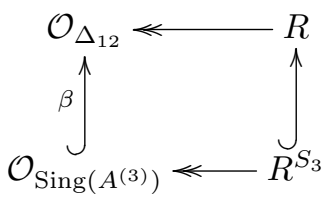

To conclude, it is enough to show that $\beta$ is not an isomorphism. Locally, $\mathcal{O}_{\Delta_{12}} \cong$ $R /\left(x_{1}-x_{2}, y_{1}-y_{2}\right)$, while by construction $\mathcal{O}_{\operatorname{Sing}\left(A^{(3)}\right)}$ is the quotient of $R^{S_{3}}$ by the homogeneous ideal $\left(x_{1}-x_{2}, y_{1}-y_{2}\right)^{S_{3}}$. Since for instance $x_{3} \in \mathcal{O}_{\Delta_{12}}$ but it is not in the image of $\beta$, the conclusion follows.

Corollary 5.7. The orbit closure $Y_{4} \subset \wedge^{3} V_{8}$ is singular along $Y_{6}$. The orbit closure $Y_{6} \subset \wedge^{3} V_{8}$ is non-normal along $Y_{8}$.

We observe that the last statement agrees with and specifies the claim in [KW] about the non-normality of $Y_{6}$.

Remark 5.8. One can observe that the isomorphism $D_{Y_{4}} \rightarrow \Sigma$ constructed in Theorem 5.2 restricts to the birational map $D_{Y_{6}} \rightarrow A$ described in Proposition 4.3. A point $x$ of $D_{Y_{6}} \backslash D_{Y_{8}}$ corresponds to $[P, P, Q]$, where $P \in \mathbf{P}\left(V_{9}^{\vee}\right)$ is the hyperplane defined by the preimage of $x$ in the desingularization of $D_{Y_{6}}$. Similarly, a point $x^{\prime} \in D_{Y_{8}}$ corresponds to $\left[P^{\prime}, P^{\prime}, P^{\prime}\right]$, where $P^{\prime} \in \mathbf{P}\left(V_{9}^{\vee}\right)$ is the hyperplane defined by the preimage of $x^{\prime}$ in the desingularization of $D_{Y_{8}}$.

\subsection{The nested Kummer fourfold and the Hilbert scheme}

Let us consider now the nested Hilbert scheme $\operatorname{Hilb}^{2,3}(A)$ parametrizing pairs $\left(S, S^{\prime}\right)$, where $S$ is a length two subscheme of $A$, and $S^{\prime} \supset S$ a length three subscheme. Such a nested Hilbert scheme is known to be smooth. Moreover, it admits an action of $A$ by translation, compatible with the sum map. So all the fibers of the latter are equivalent, and smooth. We denote them by $\operatorname{Kum}^{2,3}(A)$, the nested Kummer fourfold. By restriction from the Hilbert schemes, we get a triple cover $\operatorname{Kum}^{2,3}(A) \rightarrow \operatorname{Kum}^{2}(A)$, branched over the exceptional divisor, and also a morphism $\operatorname{Kum}^{2,3}(A) \rightarrow A$ defined by taking the residual scheme.

In our situation, $T$ is a triple cover of $D_{Y_{4}}$, which is birational to $Z \simeq$ $\operatorname{Kum}^{2}(A)$. So the fiber product of $Z$ with $T$ over $D_{Y_{4}}$ will be a triple cover of $\operatorname{Kum}^{2}(A)$, and we can expect it to be isomorphic to $\operatorname{Kum}^{2,3}(A)$. Rather than taking formally the direct product, we define $X \subset F\left(1,3,5,6,7, V_{9}\right)$ as parametrizing the flags $V_{1} \subset V_{3} \subset V_{5} \subset V_{6} \subset V_{7} \subset V_{9}$ such that $\omega$ belongs to $V_{199}+V_{359}+V_{377}+V_{666}$. Just like $T$ and $Z$, for $\omega$ generic this is a smooth fourfold. Since $V_{199}+V_{359}+V_{377}+V_{666}$ is exactly the intersection of $V_{199}+V_{369}+V_{666}$ and $V_{199}+V_{559}+V_{777}, X$ admits natural projections to $Z$ and $T$ :

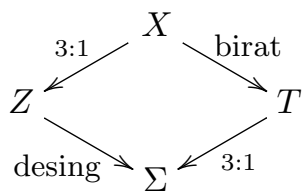


Beware that the degree three morphisms in this diagram are only generically finite. In view of Theorem 5.11, we can give a precise statement concerning $X \rightarrow Z$ :

Lemma 5.10. The positive dimensional fibers of $X \rightarrow Z$ are 81 projective lines.

Proof. We will prove that the positive dimensional fibers are 81 projective lines which are contracted to 81 points in $Z$, whose image via the desingularization $Z \rightarrow \Sigma$ is precisely $D_{Y_{8}}$. The reason behind this phenomenon is further clarified in Remark 5.13 below.

A point $z$ of $Z$ is a flag $V_{1} \subset V_{3} \subset V_{6} \subset V_{9}$ such that $\omega$ belongs to $M=$ $V_{199}+V_{369}+V_{666}$. A point $p$ of $X$ above $z$ is a pair of subspaces $\left(V_{5}, V_{7}\right)$, with $V_{1} \subset V_{3} \subset V_{5} \subset V_{6} \subset V_{7} \subset V_{9}$, such that $\omega$ belongs to $N=V_{199}+V_{359}+V_{377}+$ $V_{666}$. This is a subspace of $M$, and

$$
M / N \simeq V_{369} /\left(V_{359}+V_{169}+V_{367}\right)=V_{3} / V_{1} \otimes V_{6} / V_{5} \otimes V_{9} / V_{7} .
$$

Let $A_{2}=V_{3} / V_{1}$. The pairs $\left(V_{5}, V_{7}\right)$ are parametrized by the product of Grassmannians $\operatorname{Gr}\left(2, V_{6} / V_{3}\right) \times \operatorname{Gr}\left(1, V_{9} / V_{6}\right) \simeq \mathbf{P}^{2} \times \mathbf{P}^{2}$. On this variety, $\omega$ defines a global section of the rank four vector bundle $A_{2} \otimes V_{6} / V_{5} \otimes V_{9} / V_{7}=$ $A_{2} \otimes \mathcal{Q}_{1} \otimes \mathcal{Q}_{2}$, and the fiber over $z$ identifies with the zero locus of this section. Here we have denoted by $\mathcal{Q}_{1}$ and $\mathcal{Q}_{2}$ the quotient bundles, of rank one and two, over $\operatorname{Gr}\left(2, V_{6} / V_{3}\right)$ and $\operatorname{Gr}\left(1, V_{9} / V_{6}\right)$. An easy computation shows that $c_{4}\left(A_{2} \otimes \mathcal{Q}_{1} \otimes \mathcal{Q}_{2}\right)=c_{2}\left(\mathcal{Q}_{1} \otimes \mathcal{Q}_{2}\right)^{2}=3$, confirming that the general fiber consists in three points.

There remains to identify the infinite fibers. For this we need to analyze when a section of $A_{2} \otimes \mathcal{Q}_{1} \otimes \mathcal{Q}_{2}$ on $\operatorname{Gr}\left(2, B_{3}\right) \times \operatorname{Gr}\left(1, C_{3}\right)$ vanishes in positive dimension, where $B_{3}=V_{6} / V_{3}$ and $C_{3}=V_{9} / V_{6}$. Such a global section is an element $\gamma$ of $A_{2} \otimes B_{3} \otimes C_{3}$; as $A_{2} \otimes B_{3} \otimes C_{3}=M / V_{199}+V_{339}+V_{666}$, we have

$$
\gamma=\omega\left(\bmod V_{199}+V_{339}+V_{666}\right) .
$$

We consider $\gamma$ as a family $\Gamma$ of linear maps from $B_{3}^{\vee}$ to $C_{3}$. This section vanishes at $\left(U_{2}, U_{1}\right)$, where $U_{2} \subset B_{3}$ and $U_{1} \subset C_{3}$, if and only if $\gamma$ belongs to $A_{2} \otimes\left(U_{2} \otimes\right.$ $\left.C_{3}+B_{3} \otimes U_{1}\right)$. In other words, all the linear maps in $\Gamma$ send the line $U_{2}^{\perp}$ to the line $U_{1}$.

The classification of pencils of $3 \times 3$-matrices is well-known: there are exactly seventeen orbits (see e.g. [KW12, 5.4]). A straightforward check shows that the maximal orbits such that $\gamma$ vanishes in infinitely many pairs $\left(U_{2}, U_{1}\right)$ are those named as $\mathcal{O}_{14}, \mathcal{O}_{13}, \mathcal{O}_{11}, \mathcal{O}_{10}$, whose elements can be written respectively as follows:

i. $a_{1} \otimes b_{1} \otimes c_{1}+a_{2} \otimes b_{2} \otimes c_{2}+a_{1} \otimes b_{3} \otimes c_{3}$ for some $a_{i} \in A_{2}, b_{j} \in B_{3}, c_{k} \in C_{3}$;

ii. $a_{1} \otimes b_{1} \otimes c_{1}+a_{2} \otimes b_{2} \otimes c_{2}+a_{1} \otimes b_{2} \otimes c_{3}+a_{2} \otimes b_{3} \otimes c_{1}$ for some $a_{i} \in A_{2}$, $b_{j} \in B_{3}, c_{k} \in C_{3}$.

iii. $a_{1} \otimes b_{1} \otimes c_{1}+a_{2} \otimes b_{2} \otimes c_{2}+a_{1} \otimes b_{2} \otimes c_{3}+a_{2} \otimes b_{1} \otimes c_{3}$ for some $a_{i} \in A_{2}$, $b_{j} \in B_{3}, c_{k} \in C_{3}$;

iv. $a_{1} \otimes b_{1} \otimes c_{1}+a_{2} \otimes b_{2} \otimes c_{2}+a_{1} \otimes b_{3} \otimes c_{2}+a_{2} \otimes b_{3} \otimes c_{1}$ for some $a_{i} \in A_{2}$, $b_{j} \in B_{3}, c_{k} \in C_{3}$. 
We will show that $\omega$ being generic, $\gamma$ will never be of any of the first three types. By contradiction, we will prove that $\gamma$ being of those special types would force the class of $\omega$ modulo $V_{1}$ to belong to some orbit closure $Y$ of codimension bigger than eight in $\wedge^{3}\left(V_{9} / V_{1}\right)$. In other words, we would get a point in an ODL $D_{Y} \subset \mathbf{P}\left(V_{9}\right)$, which we know to be empty for a generic $\omega$.

i. In case i., we can find $A_{1} \subset A_{2}, B_{1} \subset B_{3}, C_{1} \subset C_{3}$ such that $\gamma$ belongs to $A_{1} \otimes B_{3} \otimes C_{3}+A_{2} \otimes B_{1} \otimes C_{1}$. This means that we can find $V_{2}, V_{4}$, $V_{7}$, with $V_{1} \subset V_{2} \subset V_{3} \subset V_{4} \subset V_{6} \subset V_{7} \subset V_{9}$ such that $\omega$ belongs to $V_{199}+V_{269}+V_{347}+V_{666}$. Modding out by $V_{1}$ and letting $U_{i}=V_{i+1} / V_{1}$, we get a point in the total space of the vector bundle $U_{158}+U_{236}+U_{555}$ over $F\left(1,2,3,5,6, U_{8}\right)$. Note that this vector bundle is a subbundle of $U_{168}+U_{666}$, which has rank 30 over the 17-dimensional flag manifold $F\left(1,6, U_{8}\right)$. So it will collapse to an orbit closure of dimension at most $30+17<48$ in $\wedge^{3} U_{8}$.

ii. In case ii., we observe that we can find $B_{1} \subset B_{3}$ and $C_{1} \subset C_{3}$ such that $\gamma$ belongs to $A_{2} \otimes B_{1} \otimes C_{3}+A_{2} \otimes B_{3} \otimes C_{1}$. This means that we can find $V_{4}, V_{7}$, with $V_{3} \subset V_{4} \subset V_{6} \subset V_{7} \subset V_{9}$ such that $\omega$ belongs to $V_{199}+V_{349}+V_{367}+V_{666}$. Modding out by $V_{1}$, we get a point in the total space of the vector bundle $U_{238}+U_{256}+U_{555}$ over $F\left(2,3,5,6, U_{8}\right)$. The latter flag manifold has dimension 25 , and the vector bundle has rank 23. But note that $U_{238}+U_{256}+U_{555} \subset U_{338}+U_{666}$, and that the vector bundle $U_{338}+U_{666}$ has rank 26 over the 21-dimensional flag manifold $F\left(3,6, U_{8}\right)$. So again it will collapse to an orbit closure of dimension at most $26+21<48$ in $\wedge^{3} U_{8}$.

iii. In case iii., there exists $B_{2} \subset B_{3}$ such that $\gamma$ belongs to $A_{2} \otimes B_{2} \otimes C_{3}$. So there exists $V_{5}$, with $V_{3} \subset V_{5} \subset V_{6}$, such that $\omega$ belongs to $V_{199}+$ $V_{359}+V_{666}$. Modding out by $V_{1}$ as before, we get a point in the total space of the vector bundle $U_{248}+U_{555}$ over $F\left(2,4,5, U_{8}\right)$. The latter flag manifold has dimension 23 , and the vector bundle has rank 25 , so it would seem to collapse to a codimension 8 orbit closure. But notice that $U_{248}+U_{555} \subset U_{448}+U_{555}$, and that now $U_{448}+U_{555}$ is a rank 28 vector bundle over $F\left(4,5, U_{8}\right)$, whose dimension is 19 . So the collapsing will have for image an orbit closure of dimension at most $28+19<48$.

So we only remain with case iv.. Observe that it occurs if and only if there exists $C_{2} \subset C_{3}$ such that $\gamma$ belongs to $A_{2} \otimes B_{3} \otimes C_{2}$. So there exists $V_{8}$, with $V_{6} \subset V_{8} \subset V_{9}$, such that $\omega$ belongs to $V_{199}+V_{339}+V_{368}+V_{666}$, and its class modulo $V_{1}$ is contained in some $U_{228}+V_{257}+U_{555}$. This is the vector bundle on $F\left(2,5,7, U_{8}\right)$ that desingularizes $Y_{8}$. In particular $V_{1}$ defines one of the 81 points of $D_{Y_{8}} \subset \mathbf{P}\left(V_{9}\right)$, the flag $V_{3} \subset V_{6} \subset V_{8}$ is uniquely defined by $V_{1}$ and there is a uniquely defined lift of $V_{1}$ in $Z$. An easy computation shows that the fiber in $X$ of this lift is a projective line, as claimed, and no further degeneration of $\omega$ can occur for $\omega$ generic.

Theorem 5.11. $X$ is isomorphic to the nested Kummer fourfold $\operatorname{Kum}^{2,3}(A)$. 
Proof. We would like to lift the isomorphism between $Z$ and $\operatorname{Kum}^{2}(A)$ :

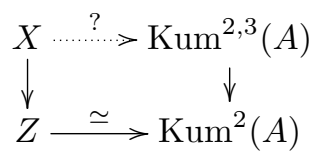

By definition, $X$ parametrizes the flags $V_{1} \subset V_{3} \subset V_{5} \subset V_{6} \subset V_{7} \subset V_{9}$ such that

$$
\omega \in V_{199}+V_{359}+V_{377}+V_{666} .
$$

Consider a hyperplane $V_{8}=\operatorname{Ker}(\phi)$ containing $V_{7}$. It defines a point in $A$ if the contraction of $\omega$ by $\phi$ has rank four. Let $v_{8}, v_{9}$ be vectors in $V_{9}$, independent modulo $V_{7}$. Modulo $V_{777}$, which is killed by $\phi$, we can write $\omega=v_{1} \wedge \alpha+\beta_{8} \wedge$ $v_{8}+\beta_{9} \wedge v_{9}$, for some $\alpha \in \wedge^{2} V_{9}$ and $\beta_{8}, \beta_{9} \in V_{3} \wedge V_{5}$. Therefore

$$
\left.\phi\lrcorner \omega=-v_{1} \wedge(\phi\lrcorner \alpha\right)+\phi\left(v_{8}\right) \beta_{8}+\phi\left(v_{9}\right) \beta_{9} .
$$

Consider the pencil $\left\langle\beta_{8}, \beta_{9}\right\rangle$. If we mod out by $V_{1} \wedge V_{5}$, we get $\left\langle\bar{\beta}_{8}, \bar{\beta}_{9}\right\rangle$ in $\wedge^{2}\left(V_{5} / V_{1}\right)$, hence in general a pencil that cuts the Grassmannian of rank two tensors along a length two subscheme. Substracting $\left.v_{1} \wedge(\phi\lrcorner \alpha\right)$ to a rank two tensor yields a tensor of rank at most four. So we get a rational map $X \rightarrow$ $\operatorname{Hilb}^{2}(A)$.

What could prevent it to be regular? First, it could happen that when we $\bmod$ out by $V_{1} \wedge V_{5}$, the pencil $\left\langle\beta_{8}, \beta_{9}\right\rangle$ collapses. In other words, $\beta_{8}$ and $\beta_{9}$ could be proportional up to $V_{1} \wedge V_{5}$. Then we may suppose that $v_{9}=0$. But this would mean that modulo $V_{1}, \omega$ depends only on seven variables, a condition that inside $\wedge^{3} \mathbb{C}^{8}$ defines an orbit of codimension $14>8$. So this cannot happen.

Second, the projected pencil $\left\langle\bar{\beta}_{8}, \bar{\beta}_{9}\right\rangle$ could be contained in the Grassmannian of rank two tensors. But then the pencil of hyperplanes that contain $V_{7}$ would be contained in $A$. Since an abelian surface cannot contain any line, this cannot happen either.

Combining the regular map $X \rightarrow \operatorname{Hilb}^{2}(A)$ with the projection $X \rightarrow Z \simeq$ $\operatorname{Kum}^{2}(A)$, we get a morphism $X \rightarrow \operatorname{Kum}^{2}(A) \times \operatorname{Hilb}^{2}(A)$ whose image is by construction contained in, hence equal to, $\operatorname{Kum}^{2,3}(A)$. Since the projections from $X$ to $Z$ and from $\operatorname{Kum}^{2,3}(A)$ to $\operatorname{Kum}^{2}(A)$ are both generically finite of degree three, we get a birational morphism from $X$ to $\operatorname{Kum}^{2,3}(A)$. But by Lemma 5.10 the exceptional locus of this birational morphism is at most onedimensional. So it has to be an isomorphism.

Remark 5.13. The positive dimensional fibers of the projection map from $\operatorname{Kum}^{2,3}(A)$ to $\operatorname{Kum}^{2}(A)$ live above the 81 three-torsion points of $A$. Indeed, if $P$ is such a point, then the fat point defined by $I(P)^{2}$ is a length three subscheme that defines a point in $\operatorname{Kum}^{2}(A)$. Since this subscheme contains all the length two schemes supported at $P$, we get a fiber isomorphic to $\mathbf{P}\left(T_{P} A\right) \simeq \mathbf{P}^{1}$. All the other length three schemes supported at $P$ are curvilinear, hence define a unique tangent. In particular we get an identification of $D_{Y_{8}}$ with $A[3]$, provided we have fixed an origin (in the preimage of $D_{Y_{8}}$ in $A$ via the map $A \rightarrow D_{Y_{6}}$ ).

Theorem 5.14. $T$ is isomorphic to $\operatorname{Hilb}^{2}(A)$.

Proof. In the proof of Theorem 5.11 we constructed a morphism $\eta_{X}: X \rightarrow$ $\mathrm{Hilb}^{2}(A)$. In fact the construction shows that this is the composition of a 
morphism from $\eta_{T}: T \rightarrow \operatorname{Hilb}^{2}(A)$ with the projection $X \rightarrow T$. Since this morphism is birational, as well as the projection $\operatorname{Kum}^{2,3}(A) \rightarrow \operatorname{Hilb}^{2}(A), \eta_{T}$ is birational. Since $T$ has trivial canonical bundle, such a birational morphism must be an isomorphism.

Remark 5.15. The group structure of $A$ allows us to define a surjective morphism $A \times \operatorname{Kum}^{1}(A)$ to $\operatorname{Hilb}^{2}(A)$ which is an étale cover of degree sixteen. This is the étale cover whose existence is predicted by the Beauville-Bogomolov decomposition.

\section{On the group structure of $A$}

In this section we geometrically describe the group structure of $A$. This is an analogue of the usual description of the group structure of a plane cubic curve from its intersection with lines. It is worth mentioning that, in a different context, Donagi [Don80] provided a geometric characterization of the group law for the $n$-dimensional abelian variety parametrizing the $(n-1)$-dimensional linear subspaces of the intersection of two general quadrics in $\mathbf{P}^{2 n+1}$, which is known to be the jacobian of a hyperelliptic curve of genus $n$.

Recall what we have established so far. If we choose two distinct points $P, Q$ of $A \subset \mathbf{P}\left(V_{9}^{\vee}\right)$, the corresponding point $z$ in $\operatorname{Hilb}^{2}(A) \simeq T$ maps to a point $\left[V_{1}\right] \in D_{Y_{4}} \subset \mathbf{P}\left(V_{9}\right)$. If this point is not on $D_{Y_{6}}$, it defines a flag $V_{1} \subset V_{3} \subset V_{6} \subset$ $V_{9}$ such that $\omega$ belongs to $V_{199}+V_{369}+V_{666}$. Moreover, its three preimages $z, z^{\prime}, z^{\prime \prime}$ in $T$ yield additional subspaces $\left(V_{5}, V_{7}\right),\left(V_{5}^{\prime}, V_{7}^{\prime}\right),\left(V_{5}^{\prime \prime}, V_{7}^{\prime \prime}\right)$, with

$$
V_{1} \subset V_{3} \subset V_{5}, V_{5}^{\prime}, V_{5}^{\prime \prime} \subset V_{6} \subset V_{7}, V_{7}^{\prime}, V_{7}^{\prime \prime} \subset V_{9},
$$

such that $\omega$ belongs to $V_{199}+V_{559}+V_{777}$, and to the corresponding spaces with $\left(V_{5}, V_{7}\right)$ replaced by $\left(V_{5}^{\prime}, V_{7}^{\prime}\right)$ and $\left(V_{5}^{\prime \prime}, V_{7}^{\prime \prime}\right)$. Since $V_{7}=P \cap Q$, this implies that if we contract $\omega$ by an equation of the hyperplane $P$ and an equation of the hyperplane $Q$, we get a vector in $V_{1}$. With a slight abuse of notation, we write

$$
[\omega(P, Q, \cdot)]=\left[V_{1}\right] \in D_{Y_{4}} \subset \mathbf{P}\left(V_{9}\right) .
$$

This yields a simple description of the map from $\operatorname{Hilb}^{2}(A) \simeq T$ to $D_{Y_{4}}$. Moreover, this is enough to characterize the point $R \in A$ such that $(P, Q, R)$ belongs to $\Sigma$ :

Proposition 6.1. Let $P, Q \in \mathbf{P}\left(V_{9}^{\vee}\right)$ be general points of $A$. Then the unique point $R \in A$ such that $(P, Q, R)$ belongs to $\Sigma$ is characterized by the condition

$$
[\omega(P, R, \cdot)]=[\omega(Q, R, \cdot)]=[\omega(P, Q, \cdot)] .
$$

Proof. The previous remarks show that $R$ verifies the required condition. There remains to prove that it is uniquely characterized by it.

Let $V_{7}:=P \cap Q$, and let us choose $v_{P} \in Q \backslash P$ and $v_{Q} \in P \backslash Q$. Let us decompose $\omega$ with respect to the direct sum $V_{9}=\mathbb{C} v_{P} \oplus \mathbb{C} v_{Q} \oplus V_{7}$, as

$$
\omega=v_{P} \wedge v_{Q} \wedge v_{1}+v_{P} \wedge \alpha+v_{Q} \wedge \beta+\sigma,
$$

with $\alpha, \beta \in \wedge^{2} V_{7}$ and $\sigma \in \wedge^{3} V_{7}$. In particular $v_{1}$ generates $V_{1}$. Since $P$ belongs to $A, v_{Q} \wedge v_{1}+\alpha$ has rank (at most) four (and since also $Q$ belongs to $A$, $v_{Q} \wedge v_{1}-\beta$ also has rank (at most) four). This means that $\alpha$ itself has rank at most four, and $v_{Q} \wedge v_{1} \wedge \alpha \wedge \alpha=0$, or equivalently $v_{1} \wedge \alpha \wedge \alpha=0$. 
Lemma 6.2. $v_{1} \wedge \alpha \wedge \alpha=0$ if and only if there exist $u, v, w$ such that $\alpha=$ $v_{1} \wedge u+v \wedge w$.

Proof. If $\alpha$ has rank six or more, then $v_{1} \wedge \alpha \wedge \alpha=0$ would imply $v_{1}=0$, which is not the case. If $\alpha$ has rank two, $\alpha \wedge \alpha=0$. So suppose that $\alpha$ has rank exactly four, which means that there exists a unique four-plane $L$ such that $\alpha$ belongs to $\wedge^{2} L$. Then $\alpha \wedge \alpha$ is a generator of $\wedge^{4} L$, and $v_{1} \wedge \alpha \wedge \alpha=0$ means that $v_{1}$ belongs to $L$. The conclusion easily follows, since if we choose a generic vector $u$ in $L$, the line generated by $\alpha$ and $v_{1} \wedge u$ in $\mathbf{P}\left(\wedge^{2} L\right)$ will meet the quadric of rank two tensors at another point.

Applying this Lemma also to $v_{Q} \wedge v_{1}-\beta$, we deduce that there exist $u, v, w, u^{\prime}, v^{\prime}, w^{\prime} \in V_{7}$ such that

$$
\omega=v_{P} \wedge v_{Q} \wedge v_{1}+v_{P} \wedge\left(v_{1} \wedge u+v \wedge w\right)+v_{Q} \wedge\left(v_{1} \wedge u^{\prime}+v^{\prime} \wedge w^{\prime}\right)+\sigma .
$$

Generically, $v_{P}, v_{Q}, v_{1}, u, v, w, u^{\prime}, v^{\prime}, w^{\prime}$ is a basis of $V_{9}$. Note that $\omega(P, R, \cdot)$ is the contraction of $v_{Q} \wedge v_{1}+v_{1} \wedge u+v \wedge w$ by $R$ (considered as a linear form). In particular it will be proportional to $v_{1}$ if and only if $v_{1}, v, w$ belong to the hyperplane $R$. Similarly $\omega(Q, R, \cdot)$ is generated by $v_{1}$ if and only if $v_{1}, v^{\prime}, w^{\prime}$ belong to $R$. So $R \supset U_{5}=\left\langle v, w, v^{\prime}, w^{\prime}, v_{1}\right\rangle$. If we let $U_{4}=\left\langle v_{P}, v_{Q}, u, u^{\prime}\right\rangle$, we are thus looking for $R \in \mathbf{P}\left(U_{4}^{\vee}\right) \cong \mathbf{P}^{3}$ such that $\omega(R, \cdot, \cdot)$ has rank four.

Let us decompose $\sigma$ further with respect to the decomposition $V_{7}=\mathbb{C} u \oplus$ $\mathbb{C} u^{\prime} \oplus U_{5}$ : there exist $\sigma_{0} \in U_{5}, \sigma_{u}, \sigma_{u^{\prime}} \in \wedge^{2} U_{5}$ and $\tilde{\sigma} \in \wedge^{3} U_{5}$ such that

$$
\sigma=u \wedge u^{\prime} \wedge \sigma_{0}+u \wedge \sigma_{u}+u^{\prime} \wedge \sigma_{u^{\prime}}+\tilde{\sigma} .
$$

As a consequence, we get

$$
\omega(R, \cdot, \cdot)=a \wedge v_{1}+b \wedge \sigma_{0}+\tau,
$$

where $a=R\left(v_{P}+u^{\prime}\right) v_{Q}+R\left(u-v_{Q}\right) v_{P}-R\left(v_{P}\right) u-R\left(v_{Q}\right) u^{\prime}$ and $b=R(u) u^{\prime}-$ $R\left(u^{\prime}\right) u$ belong to $U_{4}$, while

$$
\tau=R\left(v_{P}\right) v \wedge w+R\left(v_{Q}\right) v^{\prime} \wedge w^{\prime}+R(u) \sigma_{u}+R\left(u^{\prime}\right) \sigma_{u^{\prime}}
$$

belongs to $\wedge^{2} U_{5}$ (here again we denoted by the same letter $R$ a linear form whose kernel is the hyperplane $R$ ). If $a$ and $b$ are dependent and for example $b=0$, we need that $R(u)=R\left(u^{\prime}\right)=0$. Then $\omega(R, \cdot, \cdot)=a \wedge v_{1}+R\left(v_{P}\right) v \wedge w+R\left(v_{Q}\right) v^{\prime} \wedge w^{\prime}$ never has rank four or less, unless it is zero. The case where $b \neq 0$ is similar.

If $a$ and $b$ are independent, since generically $v_{1}$ and $\sigma_{0}$ are independent, the only way for $\omega(R, \cdot, \cdot)$ to have rank at most four is that $v_{1} \wedge \sigma_{0} \wedge \tau=0$. Since the map $v_{1} \wedge \sigma_{0} \wedge: \wedge^{2} V_{5} \longrightarrow \wedge^{4} V_{5}$ has rank three, this yields three linear conditions that determine $\left(R\left(v_{P}\right), R\left(v_{Q}\right), R(u), R\left(u^{\prime}\right)\right)$ uniquely up to scalar. So the hyperplane $R$ is uniquely determined.

The point $[\omega(P, Q, \cdot)]=\left[V_{1}\right] \in D_{Y_{4}}$ should really be thought of as the line joining $P$ and $Q$, in analogy to the line joining two points on a plane cubic, and that defines a unique third point. From this perspective, the space of "lines" is $D_{Y_{4}}$, or rather its desingularization $Z=\operatorname{Kum}^{2}(A)$.

Once we have chosen an origin $O$ of $A$, exactly as for plane cubics we can then recover the group structure on $A$ by applying Proposition 6.1 twice: starting from two general points $P, Q \in A$, we first find the point $R$ such that $(P, Q, R)$ belongs to $\Sigma$; then from the two points $O, R \in A$, we deduce the sum $P+Q \in A$. 


\section{References}

[Bea83] A. Beauville. Variétés Kähleriennes dont la première classe de Chern est nulle. J. Differential Geom., 18(4):755-782 (1984), 1983.

[Bea03] A. Beauville. The Coble hypersurfaces. C. R. Math. Acad. Sci. Paris, 337(3):189-194, 2003.

[BFMT17] V. Benedetti, S. A. Filippini, L. Manivel, and F. Tanturri. Orbital degeneracy loci and applications. 2017. To appear in Ann. Sc. Norm. Super. Pisa Cl. Sci. (5), DOI: 10.2422/2036-2145.201804_016.

[BFMT18] V. Benedetti, S. A. Filippini, L. Manivel, and F. Tanturri. Orbital degeneracy loci II: Gorenstein orbits. 2018. To appear in Int. Math. Res. Not. IMRN, DOI: 10.1093/imrn/rny272.

[BN01] M. Britze and M. A. Nieper. Hirzebruch-Riemann-Roch Formulae on Irreducible Symplectic Kähler Manifolds. 2001. Preprint ArXiv: 0101062 .

[Cob17] A. B. Coble. Point sets and allied Cremona groups. III. Trans. Amer. Math. Soc., 18(3):331-372, 1917.

[Don80] R. Donagi. Group law on the intersection of two quadrics. Ann. Scuola Norm. Sup. Pisa Cl. Sci. (4), 7(2):217-239, 1980.

[GS] D. R. Grayson and M. E. Stillman. Macaulay2, a software system for research in algebraic geometry. Available at http://www.math. uiuc. edu/Macaulay2/.

[GS15] L. Gruson and S. V. Sam. Alternating trilinear forms on a ninedimensional space and degenerations of (3,3)-polarized Abelian surfaces. Proc. Lond. Math. Soc. (3), 110(3):755-785, 2015.

[GSW13] L. Gruson, S. V. Sam, and J. Weyman. Moduli of abelian varieties, Vinberg $\theta$-groups, and free resolutions. In Commutative algebra, pages 419-469. Springer, New York, 2013.

[Gur64] G. B. Gurevich. Foundations of the theory of algebraic invariants. Translated by J. R. M. Radok and A. J. M. Spencer. P. Noordhoff Ltd., Groningen, 1964.

[Kem76] G. R. Kempf. On the collapsing of homogeneous bundles. Invent. Math., 37(3):229-239, 1976.

[KW] W. Kraskiewicz and J. Weyman. Geometry of orbit closures for the representations associated to gradings of Lie algebras of types $E_{8}$. Preprint.

[KW12] W. Kraskiewicz and J. Weyman. Geometry of orbit closures for the representations associated to gradings of Lie algebras of types $E_{6}$, $F_{4}$ and $G_{2}$. 2012. Preprint ArXiv: 1201.1102 .

[Ngu07] Q. M. Nguyen. Vector bundles, dualities and classical geometry on a curve of genus two. Internat. J. Math., 18(5):535-558, 2007. 
[NR87] M. S. Narasimhan and S. Ramanan. 20-linear systems on abelian varieties. In Vector bundles on algebraic varieties (Bombay, 1984), volume 11 of Tata Inst. Fund. Res. Stud. Math., pages 415-427. Tata Inst. Fund. Res., Bombay, 1987.

[Ort05] A. Ortega. On the moduli space of rank 3 vector bundles on a genus 2 curve and the Coble cubic. J. Algebraic Geom., 14(2):327-356, 2005 .

[RS18] E. M. Rains and S. V. Sam. Invariant theory of $\wedge^{3}(9)$ and genus-2 curves. Algebra Number Theory, 12(4):935-957, 2018.

[VE78] E. B. Vinberg and A. G. Èlašvili. A classification of the threevectors of nine-dimensional space. Trudy Sem. Vektor. Tenzor. Anal., 18:197-233, 1978. 